\title{
A CONVERGENCE RESULT FOR FINITE VOLUME SCHEMES ON RIEMANNIAN MANIFOLDS
}

\author{
JAN GIESSELMANN $^{1}$
}

\begin{abstract}
This paper studies a family of finite volume schemes for the hyperbolic scalar conservation law $u_{t}+\nabla_{g} \cdot f(x, u)=0$ on a closed Riemannian manifold $M$. For an initial value in $\mathrm{BV}(M)$ we will show that these schemes converge with a $h^{\frac{1}{4}}$ convergence rate towards the entropy solution. When $M$ is 1-dimensional the schemes are TVD and we will show that this improves the convergence rate to $h^{\frac{1}{2}}$.
\end{abstract}

Mathematics Subject Classification. 74S10, 35L65, 58J45.

Received July 30, 2008. Revised November 24, 2008.

Published online June 12, 2009.

\section{INTRODUCTION}

Hyperbolic partial differential equations on curved manifolds occur in many applications. These include shallow water models for the atmosphere or ocean $[4,12,17]$, the propagation of sound waves on curved surfaces [22] and passive tracer advection in the atmosphere. Further examples are the propagation of magneto-gravity waves in the solar tachocline $[5,9,21]$ and relativistic matter flows near compact objects like black holes $[8,15]$.

For the numerics of these problems finite difference [8], finite volume [15], discontinuous Galerkin [11] and wave propagation methods [20] have been used. Except for the work of Amorim et al. in [1] there is, up to the knowledge of the author, no convergence analysis in all of these cases. For convergence analysis of finite volume schemes, we will consider the following scalar model problem for non-linear hyperbolic conservation laws:

$$
\begin{aligned}
u_{t}+\nabla_{g} \cdot f(x, u) & =0 \text { in } M \times \mathbb{R}_{+} \\
u(x, 0) & =u_{0}(x) \text { on } M .
\end{aligned}
$$

Here $(M, g)$ is a closed oriented Riemannian manifold and $g$ is a fixed Riemannian metric on $M$. By $\nabla_{g}$. we denote the divergence operator on $M$ induced by $g$. The aim of this paper is to prove a convergence rate for finite volume schemes for this model problem.

For this problem one has the notion of entropy solution, analogous to the Kruzkov definition in Euclidean space.

\footnotetext{
Keywords and phrases. Finite volume method, conservation law, curved manifold.

${ }^{1}$ Universität Stuttgart (IANS), Pfaffenwaldring 57, 70569 Stuttgart, Germany. jan.giesselmann@mathematik. uni-stuttgart.de
} 
Definition 1.1. A function $u \in L^{\infty}\left(M \times \mathbb{R}_{+}\right)$is called an entropy solution of (1.1), (1.2) if

$$
\begin{aligned}
\int_{M \times \mathbb{R}_{+}}\left[|u-\kappa| \varphi_{t}+g(x)(f(x, u \top \kappa)-\right. & \left.\left.f(x, u \perp \kappa), \nabla_{g} \varphi\right)\right] \mathrm{d} v_{g} \mathrm{~d} t \\
& +\int_{M}\left|u_{0}-\kappa\right| \varphi(\cdot, 0) \mathrm{d} v_{g} \geq 0 \quad \forall \kappa \in \mathbb{R}, \forall \varphi \in C_{0}^{\infty}\left(M \times \mathbb{R}_{+}, \mathbb{R}_{+}\right),
\end{aligned}
$$

where $u \perp \kappa$ and $u \top \kappa$ denotes the minimum and maximum of $u$ and $\kappa$ respectively.

The well-posedness of this problem was investigated by Ben-Artzi and LeFloch in [2]. They show that given $u_{0} \in L^{\infty}(M) \cap L^{1}(M)$ and a geometry compatible flux, i.e. $\nabla_{g} \cdot f(\cdot, \bar{u})=0$, for every $\bar{u} \in \mathbb{R}$ the problem (1.1), (1.2) has a unique entropy solution $u$. Furthermore for $u_{0} \in L^{\infty}(M) \cap \mathrm{BV}(M)$ the total variation of the entropy solution is bounded for every time $t \geq 0$ in the sense that there exists $C_{1} \geq 0$ depending only on $\left\|u_{0}\right\|_{L^{\infty}(M)}$ and the geometry of $M$ such that

$$
\operatorname{TV}_{M}(u(\cdot, t)) \leq \mathrm{e}^{C_{1} t}\left(1+\mathrm{TV}_{M}\left(u_{0}\right)\right) \text { for all } t \geq 0
$$

In [1] it is shown that for a geometry compatible flux $f$ and every vector-field $X$ with $\left[X, f_{u}(\cdot, \bar{u})\right]=0$ for every $\bar{u} \in \mathbb{R}$, we have

$$
T V_{X}(u(\cdot, t)) \leq T V_{X}\left(u_{0}\right),
$$

where

$$
T V_{X}(u):=\sup _{\phi \in C \infty}(M):\|\phi\|_{L^{\infty}} \int_{M} u \nabla_{g} \cdot(\phi X) \mathrm{dv}_{g}(x) .
$$

This implies that for $d=1$ the entropy solution is total variation diminishing, i.e. $\operatorname{TV}_{M}(u(\cdot, t)) \leq T V_{M}\left(u_{0}\right)$. Furthermore they prove convergence for a class of finite volume schemes for the Cauchy-problem (1.1), (1.2). This relies on an entropy dissipation inequality for smooth entropies. We will prove a similar result for Kruzkov entropies in Lemma 5.5. In this paper we will prove convergence rates for these schemes. The general convergence framework by Eymard et al. in [7] for the proof of convergence rates for finite volume schemes in Euclidean space works but requires substantial extensions to the differential geometric framework. Particularly new problems arise in the construction and properties of cut-off functions and due to the fact that on a Riemannian manifold we cannot - in general - parallel-transport one vector to the whole manifold and get a smooth vector-field ( $c f$. the proofs of Lems. 5.6 and 5.7). As in the Euclidean case we are able to prove convergence of order $\frac{1}{2}$ in one space dimension and convergence of order $\frac{1}{4}$ in higher space dimensions.

We refer to $[19,20]$ for a treatment of the wave propagation method on curved manifolds.

We use a quite generic finite volume method. For the convergence analysis we need grids with the property that the curvature of the faces is uniformly bounded under mesh refinement and such that every point on the manifold lies only in a certain number of convex hulls of elements. In [3] different approaches to construct grids on spheres are treated and we refer to $[10,18]$ for geodesic grids on a sphere, which are an important class of examples where these results hold.

We make the following hypotheses on the data:

$$
\left\{\begin{array}{c}
u_{0} \in L^{\infty}(M) \cap \operatorname{BV}(M), U_{m}, U_{M} \in \mathbb{R}: U_{m} \leq u_{0} \leq U_{M} \text { a.e., } \\
f \in C^{1}(M \times \mathbb{R}, T M) \text { such that } f(x, \bar{u}) \in T_{x} M \text { for every } x \in M, \bar{u} \in \mathbb{R}, \\
\nabla_{g} \cdot f(\cdot, \bar{u})=0 \text { for every } \bar{u} \in \mathbb{R}, \\
\text { there is a constant } C>0 \text { such that } \\
\left\|\nabla f(x, \bar{u})-\nabla f\left(x, \bar{u}^{\prime}\right)\right\|_{g} \leq C\left|\bar{u}-\bar{u}^{\prime}\right| \text { for every } \bar{u}, \bar{u}^{\prime} \in\left[U_{m}, U_{M}\right] \text { and } x \in M .
\end{array}\right.
$$

Here $\|\cdot\|_{g}$ denotes the operator-norm induced by $g, c f$. Section $2.1, \nabla f(x, u)$ denotes the covariant derivative with respect to the first variable and $\nabla_{g} \cdot f(x, \bar{u})$ denotes the divergence which is the trace of the covariant derivative. 
The hypothesis $\operatorname{div}_{g} f(\cdot, \bar{u})=0$ is used to ensure the well-posedness of the problem and to avoid technical problems. Like in the Euclidean case it should not be necessary for the convergence rate.

The outline of this paper is as follows: in Section 2 we will recall some helpful definitions and notations from differential geometry and give some results, which are necessary for the proof of the main theorem, Theorem 5.8. In Sections 3 and 4 we will present the notion of triangulation and the construction of finite volume schemes on Riemannian manifolds respectively. In Section 5 we will state the main theorem and prove it.

\section{Differential geometry}

\subsection{Notation and definitions}

We will consider a connected, closed, oriented $d$-dimensional Riemannian manifold $(M, g)$, i.e. $M$ is a compact, smooth, oriented manifold without boundary and $g$ is a fixed Riemannian metric on $M$. This means $g(x)$ is an inner product, and hence defines a norm $\|\cdot\|_{g}$, on the tangent space $T_{x} M$ of $M$ at $x$. In local coordinates $\left(x^{j}\right)_{1 \leq j \leq d}$ the partial derivatives $\partial_{j}=\frac{\partial}{\partial x^{j}}$ form a basis of the tangent space $T_{x} M$ and we have the metric tensor $g_{i j}(x):=g(x)\left(\partial_{i}, \partial_{j}\right)$ with inverse $g^{i j}$. This enables us to define the divergence operator $\nabla_{g} \cdot$ by

$$
\nabla_{g} \cdot f(x):=\frac{1}{\sqrt{|g(x)|}} \partial_{j}\left(\sqrt{|g(x)|} f^{j}(x)\right)
$$

where $|g(x)|:=\left|\operatorname{det}\left(g_{i j}(x)\right)\right|$, for every smooth vector-field $f$ on $M$ with local representation $f=f^{j} \partial_{j}$ using the Einstein summation convention. The covariant derivative of $f$ is given by

$$
\begin{aligned}
& \nabla f: \Gamma(T M) \longrightarrow \Gamma(T M) \\
& \nabla_{Y^{j} \partial_{j}} f:=f^{i} Y^{j} \Gamma_{j i}^{k} \partial_{k}+Y^{j} \partial_{j}\left(f^{k}\right) \partial_{k},
\end{aligned}
$$

where

$$
\Gamma_{j i}^{k}:=\frac{1}{2}\left(\partial_{j} g_{i l}+\partial_{i} g_{l j}-\partial_{l} g_{j i}\right) g^{l k}
$$

and $\Gamma(T M)$ denotes the smooth vector-fields on $M$, i.e. the smooth sections of the tangent bundle $T M$. For every $x \in M$ we have

$$
\nabla f(x): T_{x} M \longrightarrow T_{x} M
$$

and by providing a norm on $T_{x} M$ the Riemannian metric defines an operator norm for such maps. The covariant derivative is compatible with the Riemannian metric, i.e. for vector-fields $X, Y, Z$ we have

$$
X(g(Y, Z))=g\left(\nabla_{X} Y, Z\right)+g\left(Y, \nabla_{X} Z\right) .
$$

These definitions of divergence and covariant derivative are only well-defined in the local coordinate system, but the definitions are independent of the choice of local coordinates and so divergence and covariant derivative are well-defined all over $M$. Similarly for every smooth function $u$ on $M$ the gradient of $u$ is defined by

$$
\left(\nabla_{g} u\right)^{i}=g^{i j} \frac{\partial u}{\partial x^{j}} .
$$

The Riemannian metric also defines a volume form $d v_{g}$ on the manifold, a volume form $d v_{N}$ on every submanifold $N$ and a metric $d_{g}$ on $M$. Spaces of functions of bounded variation are defined similar to the definition in Euclidean space. 


\section{Definition 2.1.}

$$
\begin{aligned}
\operatorname{TV}_{M}(u) & :=\sup _{X \in \Gamma(T M):\|X\|_{\infty} \leq 1} \int_{M} u \nabla_{g} \cdot X \mathrm{~d} v_{g}, \\
\operatorname{BV}(M) & :=\left\{u \in L^{1}(M): \operatorname{TV}_{M}(u)<\infty\right\} .
\end{aligned}
$$

Throughout this paper we will use the following lemma, whose proof can be found in [6] for example.

Lemma 2.2. Because $M$ is compact there exists a constant $R>0$ such that for every $x \in M$ the map $\exp _{x}^{-1}$ is a chart on $B_{4 R}(x)$.

Finally for manifolds $M, N$, a smooth map $h: M \longrightarrow N$ and $x \in M$ we will use the tangential map

$$
T h_{x}: T_{x} M \longrightarrow T_{h(x)} N
$$

which is defined by

$$
T h_{x}(v)(u):=v(h \circ u) \text { for every } v \in T_{x} M \text { and } u \in C^{\infty} N, \mathbb{R}
$$

for more details confer [6] for example.

Definition 2.3. For a set $U \subset M$ the convex hull conv $U$ of $U$ is the set of all points $x \in M$ such that there are two points $y, y^{\prime} \in M$ and a length minimizing geodesic $\gamma_{y y^{\prime}}:[0,1] \longrightarrow M$ with $\gamma_{y y^{\prime}}(0)=y, \gamma_{y y^{\prime}}(1)=y^{\prime}$ and $\gamma_{y y^{\prime}}(t)=x$ for some $t$ in $[0,1]$.

This definition is needed to define one of the properties of the grid in Definition 3.1.

\subsection{Parallel transport}

In the proof of Lemma 5.7 we will have to use parallel transport to extend vectors to local vector-fields. For $x, y \in M$ with $0<d_{g}(x, y)<R$ there exists a unique minimizing geodesic $\gamma_{x y}$ from $x$ to $y$ parametrized by arc-length. So we get a well-defined mapping

$$
P_{x y}: T_{x} M \longrightarrow T_{y} M
$$

defined by parallel transport along this geodesic. By definition of geodesic we know that $P_{x y}\left(\gamma_{x y}^{\prime}(0)\right)=$ $\gamma_{x y}^{\prime}\left(d_{g}(x, y)\right)$. Obviously we have for $0<d_{g}(x, y)<R$ the identities

$$
\nabla_{g, x} d_{g}(x, y)=-\gamma_{x y}^{\prime}(0) \quad \text { and } \quad \nabla_{g, y} d_{g}(x, y)=\gamma_{x y}^{\prime}\left(d_{g}(x, y)\right)
$$

Let $v$ be a smooth vector-field on $M$ then

$$
\frac{\mathrm{d}}{\mathrm{d} t} g\left(\gamma_{x y}(t)\right)\left(P_{x \gamma_{x y}(t)}(v(x)), \gamma_{x y}^{\prime}(t)\right)=0
$$

and therefore

$$
g(x)\left(v(x), \nabla_{g, x} d_{g}(x, y)\right)=-g(y)\left(P_{x y}(v(x)), \nabla_{g, y} d_{g}(x, y)\right)
$$

\subsection{Cut-off functions}

These are necessary for a doubling of variables argument in the proof of Lemma 5.7. Let $\psi: \mathbb{R} \longrightarrow \mathbb{R}_{+}$be a smooth function with $\operatorname{supp} \psi \subset[-1,0]$ such that

$$
\int_{\mathbb{R}} \psi(x) \mathrm{d} x=1 .
$$


Let $\psi_{\varepsilon}(x):=\frac{1}{\varepsilon} \psi\left(\frac{x}{\varepsilon}\right)$. Let $\chi: \mathbb{R} \longrightarrow \mathbb{R}_{+}$be a smooth function with support in $[-1,1]$, which is even, decreasing on $[0,1]$ and fulfils

$$
\int_{\mathbb{R}^{d}} \chi(\|x\|)=1
$$

We define

$$
\chi_{\varepsilon}: M \times M \longrightarrow \mathbb{R}, \quad(x, y) \mapsto \frac{1}{\varepsilon^{d}} \chi\left(\frac{d_{g}(x, y)}{\varepsilon}\right) .
$$

For $\varepsilon$ sufficiently small we have for every $x \in M$ using the exponential map $\exp _{x}$

$$
\int_{M} \chi_{\varepsilon}(x, y) \operatorname{dv}_{g}(x)=\int_{B_{\varepsilon}(0)} \frac{1}{\varepsilon^{d}} \chi\left(\frac{\|a\|_{g}}{\varepsilon}\right)\left|\operatorname{det}\left(T \exp _{x}^{-1}\right)_{a}\right| \mathrm{d} a .
$$

The map

$$
\left\{(x, y) \in M^{2}: d_{g}(x, y)<2 R\right\} \longrightarrow \mathbb{R} \quad(x, y) \mapsto \operatorname{det}\left(T \exp _{x}^{-1}\right) \exp _{x}^{-1}(y)
$$

is smooth and equals 1 for $x=y$. Hence we have

$$
\left|\operatorname{det}\left(T \exp _{x}^{-1}\right)_{\exp _{x}^{-1}(y)}-1\right| \leq C d_{g}(x, y)
$$

for $d_{g}(x, y) \leq R$. Inserting this in (2.6) we get

$$
\left|\int_{M} \chi_{\varepsilon}(x, y) \mathrm{dv}_{g}(y)-1\right| \stackrel{(2.4)}{\leq} \int_{B_{\varepsilon}(0)} \frac{1}{\varepsilon^{d}} \chi\left(\frac{\|a\|_{g}}{\varepsilon}\right)\left|\operatorname{det}\left(T \exp _{x}^{-1}\right)_{a}-1\right| \mathrm{d} a \leq C \varepsilon .
$$

Furthermore when $\gamma_{x y}$ denotes the length minimizing geodesic from $x$ to $y$ parametrized by arc-length, we have using (2.2)

$$
\nabla_{g, y} \chi_{\varepsilon}(x, y)=\frac{1}{\varepsilon^{d+1}} \chi^{\prime}\left(\frac{d_{g}(x, y)}{\varepsilon}\right) \gamma_{x y}^{\prime}\left(d_{g}(x, y)\right)
$$

The following technical lemma will be very helpful for the proof of Theorem 5.8. Its proof will be given in the appendix.

Lemma 2.4. There is a constant $C>0$ depending only on $M$ and $g$ such that for every $x, y \in M$ with $d_{g}(x, y)<R$ and $v \in T_{x} M$

$$
\begin{gathered}
\left|\operatorname{div}_{g, y}\left(T \exp _{x}\right)_{\exp _{x}^{-1}(y)}(v)\right| \leq C\|v\|_{g} d_{g}(x, y) \\
\left\|\left(T \exp _{x}\right)_{\exp _{x}^{-1}(y)}(v)\right\|_{g} \leq C\|v\|_{g}
\end{gathered}
$$

There is another $C>0$ such that for every $x, y \in M, v \in T_{x} M$ and $\varepsilon<R$

$$
\left|\left\langle\left(T \exp _{x}\right)_{\exp _{x}^{-1}(y)}(v)-P_{x y}(v), \nabla_{g, y} \chi_{\varepsilon}(x, y)\right\rangle\right| \leq \frac{C}{\varepsilon^{d-1}}\|v\|_{g} 1_{\left\{d_{g}(x, y)<\varepsilon\right\}} .
$$

Here we have $\left(T \exp _{x}\right)_{\exp _{x}^{-1}(y)}(v) \in T_{y} M$ for every $y \in B_{R}(x)$ and hence

$$
y \mapsto\left(T \exp _{x}\right)_{\exp _{x}^{-1}(y)}(v)
$$

defines a vector-field on $B_{R}(x)$. 


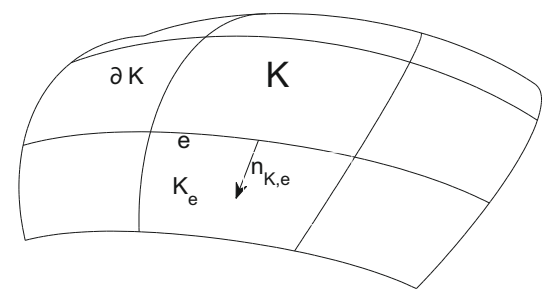

Figure 1. Part of a triangulation of the sphere.

\section{Triangulation}

Definition 3.1. A curved polyhedron $K$ is a closed subset of $M$, such that the boundary of $K$ is the union of finitely many hypersurfaces with boundary $e$ of $M$, which are called the faces of $K$, satisfying $e_{1} \cap e_{2}$ is empty or a submanifold of $M$ with dimension at most $d-2$. Furthermore we impose $\overline{K^{\circ}}=K$ to rule out some forms of degeneracy of $K$. A triangulation on $(M, g)$ is a set $\mathcal{T}$ of curved polyhedra $K$ on $M$, such that $M=\cup_{\mathcal{T}} \bar{K}$. We impose $K_{1} \cap K_{2}$ is a common face of $K_{1}, K_{2}$ or a submanifold of dimension $\leq d-2$.

The set of the faces $e$ of a polyhedron $K$ is denoted by $\partial K$ and the unique polyhedron sharing the face $e$ with $K$ is denoted by $K_{e}$. By $n_{K, e}(x) \in T_{x} M$ we denote the unit outer normal to a polyhedron $K$ in a point $x \in e$. These definitions are illustrated in Figure 1. Finally $|K|,|e|$ denote the $d$ - and $(d-1)$-dimensional Hausdorff measures of $K$ and $e$ respectively.

We will need the following assumption on the triangulation: There exist $C, \bar{R}, \beta, h>0$ and $k, N_{c} \in \mathbb{N}$ such that for every $K \in \mathcal{T}$ and $e \in \partial K$ the following conditions are fulfilled

$$
\begin{aligned}
\beta h^{d} & \leq|K|, \\
|e| & \leq C h^{d-1}, \\
\# \partial K & \leq k, \\
\delta(K) & \leq h, \\
\sup _{x, i, e}\left|\lambda_{i}(e)(x)\right| & \leq \bar{R}, \\
\sup _{x \in M} \#\{K: x \in \operatorname{conv} K\} & \leq N_{c},
\end{aligned}
$$

where $\delta(K):=\sup \left\{d_{g}(x, y): x, y \in K\right\}$ and \# denotes the number of elements of a certain set. Furthermore $\lambda_{i}(e)(x)$ denotes the $i$ th principal curvature of the face $e$ in the point $x \in e$.

Note. The condition (3.6) should be fulfilled for all computational meshes and is needed for the approximation of the initial data in Lemma 3.2 which will be stated below and whose proof can be found in the appendix. Under mesh refinement condition (3.5) is satisfied for example by geodesic grids and the combined grid composed of a latitude-longitude grid away from the poles and a stereographic grid at the two polar caps which can be found in [14]. It is not satisfied by the latitude-longitude grid on the sphere which also has some other numerical drawbacks. In particular strongly differing cell sizes impose hard CFL conditions on the timestep (cf. [3]). The grids proposed in [3] also do not satisfy this condition.

Lemma 3.2. For $h$ small enough and every $u \in \mathrm{BV}(M)$ there is a constant $C>0$ depending on $M$ and $N_{c}$ such that

$$
\|u-\bar{u}\|_{L^{1}(M)} \leq C h,
$$


where

so $\bar{u}$ is well-defined almost everywhere on the manifold.

$$
\bar{u}(x):=\frac{1}{|K|} \int_{K} u(x) \mathrm{dv}_{g}(x) \text { for } x \in K,
$$

\section{THE SCHEME}

For every polyhedron $K \in \mathcal{T}$ and face $e \in \partial K$ we consider a numerical flux function $f_{K, e}: \mathbb{R} \times \mathbb{R} \longrightarrow \mathbb{R}$ such that the following properties are satisfied:

$$
\begin{aligned}
& \text { Conservation: } \quad f_{K, e}(a, b)=-f_{K_{e}, e}(b, a), \\
& \text { Consistency: } f_{K, e}(a, a)= \frac{1}{|e|} \int_{e} f(x, a) n_{K, e}(x) \mathrm{dv}_{e}(x), \\
& \text { Monotonicity: } \quad f_{K, e} \quad \text { is nondecreasing in the first and } \\
& \text { nonincreasing in the second variable. }
\end{aligned}
$$

Furthermore we impose that the $f_{K, e}$ are uniformly locally Lipschitz continuous. We will consider the following semi-discrete scheme:

$$
\begin{aligned}
\left(u_{K}^{h}\right)_{t} & =-\frac{1}{|K|} \sum_{e \in \partial K}|e| f_{K, e}\left(u_{K}^{h}, u_{K_{e}}^{h}\right) \\
u_{K}^{h}(0) & =\frac{1}{|K|} \int_{K} u_{0}(x) \mathrm{dv}_{g}(x) \\
u^{h}(x, t) & =u_{K}^{h}(t) \text { for } x \in K .
\end{aligned}
$$

\section{Proof of COnVergence Rates}

We first show that a solution of (4.4)-(4.6) exists and that it is bounded.

Lemma 5.1. Assume the local existence of a solution of (4.4)-(4.6) and let $u_{0}(x) \in\left[U_{m}, U_{M}\right]$ for almost every $x \in M$, then $u_{K}^{h}(t) \in\left[U_{m}, U_{M}\right]$ for every $t \geq 0$ and $K \in \mathcal{T}$.

Proof. It is obvious that $u_{K}^{h}(0) \in\left[U_{m}, U_{M}\right]$ for every $K$. First observe that for fixed $K$ and $u_{K_{e}}^{h} \leq u_{K}^{h}$ for all $e \in \partial K$ we have

$$
\begin{aligned}
\left(u_{K}^{h}\right)_{t} & =-\frac{1}{|K|} \sum_{e \in \partial K}|e| f_{K, e}\left(u_{K}^{h}, u_{K_{e}}^{h}\right) \\
\stackrel{\text { monotonicity }}{\leq} & -\frac{1}{|K|} \sum_{e \in \partial K}|e| f_{K, e}\left(u_{K}^{h}, u_{K}^{h}\right) \\
\stackrel{\text { consistency }}{\leq} & -\frac{1}{|K|} \sum_{e \in \partial K} \int_{e} f\left(x, u_{K}^{h}\right) n_{K, e} \mathrm{dv}_{g}(x)=0 .
\end{aligned}
$$

As an immediate consequence of Lemma 5.1 and the local Lipschitz continuity of the numerical fluxes we have:

Corollary 5.2. There exists a global solution of the system (4.4)-(4.6).

The next step is to prove a TVD estimate in the $d=1$ case and a weak BV-estimate in the $d \geq 2$ case. For brevity we introduce the following notation: for real numbers $a, b$ we define

$$
C(a, b):=\left\{(c, d) \in[a \perp b, a \top b]^{2}:(b-a)(d-c) \geq 0\right\},
$$


where $a \top b$ and $a \perp b$ denote the maximum and minimum of $a$ and $b$ respectively. For every $t \geq 0$ we define

$$
E(t):=\left\{(K, e): K \in \mathcal{T}, e \in \partial K, u_{K}^{h}(t)>u_{K_{e}}^{h}(t)\right\} .
$$

Lemma 5.3 (TVD property). Let $M$ be 1-dimensional then the scheme (4.4)-(4.6) is TVD, i.e.

$$
\operatorname{TV}_{M}\left(u^{h}(\cdot, t)\right) \leq \operatorname{TV}_{M}\left(u_{0}\right) \text { for all } t>0 .
$$

This implies that for every $T>0$ there exists a $C>0$ depending only on $f, u_{0}, M, f_{K, e}$, T such that

$$
\int_{0}^{T} \sum_{K \in \mathcal{T}} \sum_{e \in \partial K}|e| \max _{(c, d) \in C\left(u_{K}^{h}, u_{K_{e}}^{h}\right)}\left|f_{K, e}(c, d)-f_{K, e}(c, c)\right| \mathrm{d} t \leq C .
$$

Proof. We will consider times $t$ where $\frac{\mathrm{d}}{\mathrm{d} t}\left|u_{K}^{h}-u_{K_{e}}^{h}\right|$ exists for all $K \in \mathcal{T}$ and $e \in \partial K$. These derivatives exist for almost every $t \geq 0$ and we have

$$
\frac{\mathrm{d}}{\mathrm{d} t} \operatorname{TV}_{M}\left(u^{h}(\cdot, t)\right)=\frac{1}{2} \sum_{K \in \mathcal{T}} \sum_{e \in \partial K} \frac{\mathrm{d}}{\mathrm{d} t}\left|u_{K}^{h}(t)-u_{K_{e}}^{h}(t)\right| .
$$

Now we fix one $K \in \mathcal{T}$ and observe that $K$ has exactly two neighbours $K_{1}, K_{2}$.

- If $u_{K_{1}}^{h}(t) \leq u_{K}^{h}(t) \leq u_{K_{2}}^{h}(t)$ or $u_{K_{2}}^{h}(t) \leq u_{K}^{h}(t) \leq u_{K_{1}}^{h}(t)$ then $\left(u_{K}^{h}\right)_{t}$ occurs exactly twice with different signs in the sum and therefore vanishes.

- If $u_{K}^{h}(t)>u_{K_{1}}^{h}(t), u_{K_{2}}^{h}(t)$ the term

$$
\left(u_{K}^{h}\right)_{t}(t)=-\sum_{e \in \partial K} \frac{|e|}{|K|} f_{K, e}\left(u_{K}^{h}(t), u_{K_{e}}^{h}(t)\right) \leq-\sum_{e \in \partial K} \frac{|e|}{|K|} f_{K, e}\left(u_{K}^{h}(t), u_{K}^{h}(t)\right)=0
$$

occurs twice in the sum.

- If $u_{K}^{h}(t)<u_{K_{1}}^{h}(t), u_{K_{2}}^{h}(t)$ the term

$$
-\left(u_{K}^{h}\right)_{t}(t)=\sum_{e \in \partial K} \frac{|e|}{|K|} f_{K, e}\left(u_{K}^{h}(t), u_{K_{e}}^{h}(t)\right) \leq \sum_{e \in \partial K} \frac{|e|}{|K|} f_{K, e}\left(u_{K}^{h}(t), u_{K}^{h}(t)\right)=0
$$

occurs twice in the sum.

So we know $\operatorname{TV}_{M}\left(u^{h}(\cdot, t)\right)$ is nonincreasing in time. For every $K \in \mathcal{T}$ there exist $x_{K}, y_{K} \in K$ such that

$$
u_{0}\left(x_{K}\right) \geq u_{K}^{h}(0) \geq u_{0}\left(y_{K}\right) .
$$

Let $K_{1}, K_{2}$ be the neighboring elements for some $K \in \mathcal{T}$, then we define

$$
\zeta_{K}= \begin{cases}x_{K}: & u_{K}^{h}>u_{K_{1}}^{h}, u_{K_{2}}^{h} \\ y_{K}: & \text { else. }\end{cases}
$$

We have

$$
\begin{aligned}
2 \operatorname{TV}_{M}\left(u^{h}(\cdot, 0)\right) & =\sum_{K \in \mathcal{T}} \sum_{e \in \partial K}\left|u_{K}^{h}(0)-u_{K_{e}}^{h}(0)\right| \\
& \leq \sum_{K \in \mathcal{T}} \sum_{e \in \partial K}\left|u_{0}\left(\zeta_{K}\right)-u_{0}\left(\zeta_{K_{e}}\right)\right| \leq 2 \operatorname{TV}_{M}\left(u_{0}\right) .
\end{aligned}
$$


This proves the TVD property. For $(c, d) \in C\left(u_{K}^{h}, u_{K_{e}}^{h}\right)$ we have

$$
\left|f_{K, e}(c, d)-f_{K, e}(c, c)\right| \leq L|c-d| \leq L\left|u_{K}^{h}-u_{K_{e}}^{h}\right|,
$$

where $L$ is the uniform Lipschitz constant for all $f_{K, e}$ on $\left[U_{m}, U_{M}\right]$. Using $|e|=2$ we get

$$
\begin{aligned}
\int_{0}^{T} \sum_{K \in \mathcal{T}} \sum_{e \in \partial K}|e| \max _{(c, d) \in C\left(u_{K}^{h}, u_{K_{e}}^{h}\right)}\left|f_{K, e}(c, d)-f_{K, e}(c, c)\right| \mathrm{d} t & \leq \int_{0}^{T} 2 L \sum_{K \in \mathcal{T}} \sum_{e \in \partial K}\left|u_{K}^{h}-u_{K_{e}}^{h}\right| \mathrm{d} t \\
& \leq 4 L \int_{0}^{T} \operatorname{TV}_{M}\left(u^{h}(\cdot, t)\right) \mathrm{d} t \leq 4 L T \operatorname{TV}_{M}\left(u_{0}\right)
\end{aligned}
$$

In the higher-dimensional case there is no TVD estimate, but we can prove a weak BV estimate which will play a similar role in the convergence proof.

Lemma 5.4 (weak BV-estimate). Let $d \geq 2$ be the dimension of $M$. For every $T>0$ there exists $C>0$ depending only on $f, u_{0}, M, \beta,\left\{f_{K, e}\right\}, T, k$ such that

$$
\int_{0}^{T} \sum_{K \in \mathcal{T}} \sum_{e \in \partial K}|e| \max _{(c, d) \in C\left(u_{K}^{h}, u_{K_{e}}^{h}\right)}\left|f_{K, e}(c, d)-f_{K, e}(c, c)\right| \mathrm{d} t \leq \frac{C}{\sqrt{h}} .
$$

Proof. We have

$$
\begin{aligned}
\int_{0}^{T} \sum_{K \in \mathcal{T}}|K| u_{K}^{h}\left(u_{K}^{h}\right)_{t} \mathrm{~d} t & =\frac{1}{2} \int_{0}^{T} \sum_{K \in \mathcal{T}}|K|\left(\left(u_{K}^{h}\right)^{2}\right)_{t} \mathrm{~d} t \\
& =\frac{1}{2} \sum_{K \in \mathcal{T}}|K|\left(\left(u_{K}^{h}\right)^{2}(T)-\left(u_{K}^{h}\right)^{2}(0)\right) \\
& \geq-\frac{1}{2} \sum_{K \in \mathcal{T}}|K|\left(u_{K}^{h}\right)^{2}(0) \\
& =-\frac{1}{2}\left\|u^{h}(0)\right\|_{L^{2}(M)}^{2} \geq-\frac{1}{2}\left\|u_{0}\right\|_{L^{2}(M)}^{2} .
\end{aligned}
$$

Now we multiply (4.4) by $|K| u_{K}^{h}(t)$ and sum over all $K \in \mathcal{T}$

$$
\begin{aligned}
\int_{0}^{T} \sum_{K \in \mathcal{T}}|K| u_{K}^{h}\left(u_{K}^{h}\right)_{t} \mathrm{~d} t= & -\int_{0}^{T} \sum_{K \in \mathcal{T}} \sum_{e \in \partial K}|e| f_{K, e}\left(u_{K}^{h}, u_{K_{e}}^{h}\right) u_{K}^{h} \mathrm{~d} t \\
\stackrel{(4.2)(1.4)}{=} & \int_{0}^{T} \sum_{K \in \mathcal{T}} \sum_{e \in \partial K}|e|\left(f_{K, e}\left(u_{K}^{h}, u_{K}^{h}\right)-f_{K, e}\left(u_{K}^{h}, u_{K_{e}}^{h}\right)\right) u_{K}^{h} \mathrm{~d} t \\
= & \int_{0}^{T} \sum_{(K, e) \in E(t)}|e|\left[\left(f_{K, e}\left(u_{K}^{h}, u_{K}^{h}\right)-f_{K, e}\left(u_{K}^{h}, u_{K_{e}}^{h}\right)\right) u_{K}^{h}\right. \\
& \left.+\left(f_{K_{e}, e}\left(u_{K_{e}}^{h}, u_{K_{e}}^{h}\right)-f_{K_{e}, e}\left(u_{K_{e}}^{h}, u_{K}^{h}\right)\right) u_{K_{e}}^{h}\right] \mathrm{d} t \\
\stackrel{(4.1)}{=} & \int_{0}^{T} \sum_{(K, e) \in E(t)}|e|\left[\left(f_{K, e}\left(u_{K}^{h}, u_{K}^{h}\right)-f_{K, e}\left(u_{K}^{h}, u_{K_{e}}^{h}\right)\right) u_{K}^{h}\right. \\
& \left.-\left(f_{K, e}\left(u_{K_{e}}^{h}, u_{K_{e}}^{h}\right)-f_{K, e}\left(u_{K}^{h}, u_{K_{e}}^{h}\right)\right) u_{K_{e}}^{h}\right] \mathrm{d} t .
\end{aligned}
$$


Now we define $F_{K, e}(a):=f_{K, e}(a, a)$ and let $\Phi_{K, e}$ be a primitive of $a \mapsto a F_{K, e}^{\prime}(a)$ satisfying $\Phi_{K, e}(0)=0$. Let $a=u_{K}^{h}, b=u_{K_{e}}^{h}$ then every single summand has the form

$$
|e|\left[a\left(F_{K, e}(a)-f_{K, e}(a, b)\right)-b\left(F_{K, e}(b)-f_{K, e}(a, b)\right)\right] .
$$

Integration by parts yields

$$
\begin{aligned}
\Phi_{K, e}(b)-\Phi_{K, e}(a) & =\int_{a}^{b} u F_{K, e}^{\prime}(u) \mathrm{d} u \\
& =b\left(F_{K, e}(b)-f_{K, e}(a, b)\right)-a\left(F_{K, e}(a)-f_{K, e}(a, b)\right)-\int_{a}^{b}\left(F_{K, e}(u)-f_{K, e}(a, b)\right) \mathrm{d} u .
\end{aligned}
$$

Due to the conservation property (4.1) of the numerical fluxes we have $F_{K, e}=-F_{K_{e}, e}$ and therefore $\Phi_{K, e}=$ $-\Phi_{K_{e}, e}$. Because the flux is geometry compatible (1.4) we have

$$
\sum_{e \in \partial K}|e| f_{K, e}(a, a)=0 \Longrightarrow \sum_{e \in \partial K}|e| F_{K, e}^{\prime}(a)=0 \Longrightarrow \sum_{e \in \partial K}|e| \Phi_{K, e}(a)=0
$$

for every $K \in \mathcal{T}$ and $a \in \mathbb{R}$. Thus we have

$$
\begin{aligned}
\sum_{(K, e) \in E(t)}|e|\left(\Phi_{K, e}\left(u_{K}^{h}\right)-\Phi_{K, e}\left(u_{K_{e}}^{h}\right)\right)= & \sum_{(K, e) \in E(t)}|e|\left(\Phi_{K, e}\left(u_{K}^{h}\right)+\Phi_{K_{e}, e}\left(u_{K_{e}}^{h}\right)\right) \\
= & \sum_{(K, e) \in E(t)}|e|\left(\Phi_{K, e}\left(u_{K}^{h}\right)+\Phi_{K_{e}, e}\left(u_{K_{e}}^{h}\right)\right) \\
& +\underbrace{\sum_{\left\{(K, e): u_{K}^{h}=u_{K_{e}}^{h}\right\}}|e|\left(\Phi_{K, e}\left(u_{K}^{h}\right)\right)}_{0} \\
= & \sum_{K \in \mathcal{T}} \sum_{e \in \partial K}|e| \Phi_{K, e}\left(u_{K}^{h}\right)=0 .
\end{aligned}
$$

Using this in (5.2) implies

$$
\begin{aligned}
\int_{0}^{T} \sum_{K \in \mathcal{T}}|K| u_{K}^{h}\left(u_{K}^{h}\right)_{t} \mathrm{~d} t & =-\int_{0}^{T} \sum_{K \in \mathcal{T}} \sum_{e \in \partial K}|e| f_{K, e}\left(u_{K}^{h}, u_{K_{e}}^{h}\right) u_{K}^{h} \mathrm{~d} t \\
& =-\int_{0}^{T} \sum_{(K, e) \in E(t)}|e| \int_{u_{K}^{h}}^{u_{K_{e}}^{h}}\left(f_{K, e}(u, u)-f_{K, e}\left(u_{K}^{h}, u_{K_{e}}^{h}\right)\right) \mathrm{d} u \mathrm{~d} t \\
& =\int_{0}^{T} \sum_{(K, e) \in E(t)}|e| \int_{u_{K e}^{h}}^{u_{K}^{h}}\left(f_{K, e}(u, u)-f_{K, e}\left(u_{K}^{h}, u_{K_{e}}^{h}\right)\right) \mathrm{d} u \mathrm{~d} t .
\end{aligned}
$$

For $u_{K_{e}}^{h} \leq c \leq d \leq u_{K}^{h}$ we have due to (4.3)

$$
\begin{aligned}
\int_{u_{K_{e}}^{h}}^{u_{K}^{h}} \underbrace{\left(f_{K, e}\left(u_{K}^{h}, u_{K_{e}}^{h}\right)-f_{K, e}(u, u)\right)}_{\geq 0} \mathrm{~d} u & \geq \int_{c}^{d}\left(f_{K, e}\left(u_{K}^{h}, u_{K_{e}}^{h}\right)-f_{K, e}(u, u)\right) \mathrm{d} u \\
& \geq \int_{c}^{d}\left(f_{K, e}(d, c)-f_{K, e}(u, u)\right) \mathrm{d} u
\end{aligned}
$$


For a monotonic Lipschitz continuous function $g: \mathbb{R} \longrightarrow \mathbb{R}$, with Lipschitz constant $G>0$ we have

$$
\left|\int_{c}^{d}(g(u)-g(c)) \mathrm{d} u\right| \geq \frac{1}{2 G}(g(d)-g(c))^{2}, \forall c, d \in \mathbb{R}
$$

which can be seen by the substitution $v=g(u)-g(c)$. A similar estimate is used in [7].

Thus (4.3) and the Lipschitz continuity of the $f_{K, e}$ imply

$$
\begin{aligned}
\int_{c}^{d}\left(f_{K, e}(d, c)-f_{K, e}(u, u)\right) \mathrm{d} u & \geq \int_{c}^{d}\left(f_{K, e}(d, c)-f_{K, e}(d, u)\right) \mathrm{d} u \\
& \geq \frac{1}{2 L}\left(f_{K, e}(d, c)-f_{K, e}(d, d)\right)^{2} \\
\text { and } & \\
\int_{c}^{d}\left(f_{K, e}(d, c)-f_{K, e}(u, u)\right) \mathrm{d} u & \geq \int_{c}^{d}\left(f_{K, e}(d, c)-f_{K, e}(u, c)\right) \mathrm{d} u \\
& \geq \frac{1}{2 L}\left(f_{K, e}(d, c)-f_{K, e}(c, c)\right)^{2},
\end{aligned}
$$

where $L$ is the uniform Lipschitz constant of the $f_{K, e}$ on $\left[U_{m}, U_{M}\right]$. Multiplying both inequalities with $\frac{1}{2}$ and adding them yields with (5.1) and (5.3)

$$
\begin{aligned}
\frac{1}{2}\left\|u_{0}\right\|_{L^{2}(M)}^{2} \geq & \int_{0}^{T} \sum_{(K, e) \in E(t)} \int_{u_{K_{e}}^{h}}^{u_{K}^{h}}|e|\left(f_{K, e}\left(u_{K}^{h}, u_{K_{e}}^{h}\right)-f_{K, e}(u, u)\right) \mathrm{d} u \mathrm{~d} t \\
\geq & \int_{0}^{T} \sum_{(K, e) \in E(t)} \frac{|e|}{2 L}\left(\max _{u_{K_{e}}^{h} \leq c \leq d \leq u_{K}^{h}}\left(f_{K, e}(d, c)-f_{K, e}(d, d)\right)^{2}\right. \\
& \left.+\max _{u_{K_{e}}^{h} \leq c \leq d \leq u_{K}^{h}}\left(f_{K, e}(d, c)-f_{K, e}(c, c)\right)^{2}\right) \mathrm{d} t \\
\geq & \int_{0}^{T} \sum_{K \in \mathcal{T}} \sum_{e \in \partial K} \frac{|e|}{2 L} \max _{(c, d) \in C\left(u_{K}^{h}, u_{K_{e}}^{h}\right)}\left|f_{K, e}(c, d)-f_{K, e}(c, c)\right|^{2} \mathrm{~d} t .
\end{aligned}
$$

Now by Cauchy Schwartz inequality we get

$$
\begin{aligned}
& \int_{0}^{T} \sum_{K \in \mathcal{T}} \sum_{e \in \partial K}|e| \max _{(c, d) \in C\left(u_{K}^{h}, u_{K_{e}}^{h}\right)}\left|f_{K, e}(c, d)-f_{K, e}(c, c)\right| \mathrm{d} t \\
& \leq\left(\int_{0}^{T} \sum_{K \in \mathcal{T}} \sum_{e \in \partial K}|e|^{2} \max _{(c, d) \in C\left(u_{K}^{h}, u_{K e}^{h}\right)}\left|f_{K, e}(c, d)-f_{K, e}(c, c)\right|^{2} \mathrm{~d} t\right)^{\frac{1}{2}} \cdot\left(\sum_{K \in \mathcal{T}} \sum_{e \in \partial K} 1\right)^{\frac{1}{2}} \\
& \leq C L^{\frac{1}{2}}\left\|u_{0}\right\|_{L^{2}(M)} h^{\frac{d-1}{2}} h^{-\frac{d}{2}} \frac{1}{\beta^{\frac{1}{2}}} k^{\frac{1}{2}},
\end{aligned}
$$

the last line follows from (5.4) and the assumptions on the grid (3.1)-(3.3).

Next we prove a weak discrete entropy inequality for the approximate solution, which is an auxiliary result to prove a continuous entropy inequality for the approximate solution. This continuous entropy inequality is important for the main convergence proof and has a similar importance for the proof like the entropy inequality for the exact solution. 
Lemma 5.5 (weak discrete entropy inequality). For every $\kappa \in\left[U_{m}, U_{M}\right]$, every polyhedron $K \in \mathcal{T}$ and every test function $\varphi \in C_{0}^{\infty}\left(\mathbb{R}_{+}, \mathbb{R}_{+}\right)$the following inequality holds

$$
\begin{aligned}
\int_{\mathbb{R}_{+}}|K|\left|u_{K}^{h}(t)-\kappa\right| \varphi_{t} \mathrm{~d} t+|K|\left|u_{K}^{h}(0)-\kappa\right| \varphi(0) & \\
& -\int_{\mathbb{R}_{+}} \sum_{e \in \partial K}|e|\left(f_{K, e}\left(u_{K}^{h} \top \kappa, u_{K_{e}}^{h} \top \kappa\right)-f_{K, e}\left(u_{K}^{h} \perp \kappa, u_{K_{e}}^{h} \perp \kappa\right)\right) \varphi \mathrm{d} t \geq 0 .
\end{aligned}
$$

Proof. Let $B=\sup \{t>0: \varphi(t) \neq 0\}$. Consider disjoint intervals $\left\{I_{j}=\left(a_{j}, b_{j}\right): j \in \mathcal{J}\right\}$, where $\mathcal{J}$ is some countable index set, such that

$$
A:=\bigcup_{j \in \mathcal{J}} I_{j}=\left\{t \in(0, B): u_{K}^{h}(t)>\kappa\right\}
$$

For all $b_{j}$ we have $u_{K}^{h}\left(b_{j}\right)=\kappa$ or $\varphi\left(b_{j}\right)=0$. For all but at most one $a_{j}$ we have $u_{K}^{h}\left(a_{j}\right)=\kappa$. If there is an $a_{*} \in\left\{a_{j}: j \in \mathcal{J}\right\}$ with $u_{K}^{h}\left(a_{*}\right) \neq \kappa$ we have $a_{*}=0$. To make the proof shorter we nevertheless denote one $a_{j}$ by $a_{*}$ satisfying $a_{*}=0$ or $u_{K}^{h}\left(a_{*}\right)=\kappa$. Using this notation we have

$$
\begin{aligned}
|K| \int_{\mathbb{R}_{+}}\left(u_{K}^{h}(t) \top \kappa\right) \varphi_{t} \mathrm{~d} t & =|K| \sum_{j} \int_{I_{j}} u_{K}^{h} \varphi_{t} \mathrm{~d} t+|K| \int_{\mathbb{R}_{+} \backslash A} \kappa \varphi_{t} \mathrm{~d} t \\
& =|K| \sum_{j} \int_{I_{j}}\left(u_{K}^{h}-\kappa\right) \varphi_{t} \mathrm{~d} t+|K| \int_{\mathbb{R}_{+}} \kappa \varphi_{t} \mathrm{~d} t \\
& =|K|\left(\sum_{j}\left[\left(u_{K}^{h}-\kappa\right)\left(b_{j}\right) \varphi\left(b_{j}\right)-\left(u_{K}^{h}-\kappa\right)\left(a_{j}\right) \varphi\left(a_{j}\right)-\int_{I_{j}}\left(u_{K}^{h}\right)_{t} \varphi \mathrm{d} t\right]-\kappa \varphi(0)\right) \\
& =-|K| u_{K}^{h}\left(a_{*}\right) \varphi\left(a_{*}\right)+\kappa|K|\left(\varphi\left(a_{*}\right)-\varphi(0)\right)-\int_{A}|K|\left(u_{K}^{h}\right)_{t} \varphi \mathrm{d} t \\
& \geq-\left(u_{K}^{h}(0) \top \kappa\right) \varphi(0)|K|-\int_{A}|K|\left(u_{K}^{h}\right)_{t} \varphi \mathrm{d} t .
\end{aligned}
$$

For $t \in A$ we have by (4.3) and (4.4)

$$
\begin{aligned}
|K|\left(u_{K}^{h}\right)_{t} \varphi & =-\sum_{e \in \partial K}|e| f_{K, e}\left(u_{K}^{h} \top \kappa, u_{K_{e}}^{h}\right) \varphi \\
& \leq-\sum_{e \in \partial K}|e| f_{K, e}\left(u_{K}^{h} \top \kappa, u_{K_{e}}^{h} \top \kappa\right) \varphi
\end{aligned}
$$

while for $t \in \mathbb{R} \backslash A$ we have by (1.4), (4.2) and (4.3)

$$
\begin{aligned}
0 & =\varphi \sum_{e \in \partial K}|e| f_{K, e}\left(u_{K}^{h} \top \kappa, \kappa\right) \\
& \leq-\sum_{e \in \partial K}|e| f_{K, e}\left(u_{K}^{h} \top \kappa, u_{K_{e}}^{h} \top \kappa\right) \varphi .
\end{aligned}
$$

Thus we get

$$
|K| \int_{\mathbb{R}_{+}}\left(u_{K}^{h} \top \kappa\right) \varphi_{t} \mathrm{~d} t+\left(u_{K}^{h}(0) \top \kappa\right) \varphi(0)|K|-\int_{\mathbb{R}_{+}} \sum_{e \in \partial K}|e| f_{K, e}\left(u_{K}^{h} \top \kappa, u_{K_{e}}^{h} \top \kappa\right) \varphi \mathrm{d} t \geq 0 .
$$


In a similar way we can prove

$$
|K| \int_{\mathbb{R}_{+}}\left(u_{K}^{h} \perp \kappa\right) \varphi_{t} \mathrm{~d} t+\left(u_{K}^{h}(0) \perp \kappa\right) \varphi(0)|K|-\int_{\mathbb{R}_{+}} \sum_{e \in \partial K}|e| f_{K, e}\left(u_{K}^{h} \perp \kappa, u_{K_{e}}^{h} \perp \kappa\right) \varphi \mathrm{d} t \leq 0 .
$$

The lemma follows from $\left|u_{K}^{h}(t)-\kappa\right|=\left(u_{K}^{h}(t) \top \kappa\right)-\left(u_{K}^{h}(t) \perp \kappa\right)$.

We observe that because $M$ is compact (1.4) implies that the norms $\|f\|_{L^{\infty}(M)}$ and $\|\nabla f\|_{g}$ are bounded by a constant $C_{2}$. This means particularly for every unit vector $t$ tangent to $M$ the following estimate for the covariant derivative in direction $t$ holds: $\left\|\nabla_{t} f\right\|_{g} \leq C_{2}$ on $M \times\left[U_{m}, U_{M}\right]$.

Lemma 5.6 (continuous entropy inequality). Provided the assumptions (3.1)-(3.4) on the grid with $h$ small enough and (4.1)-(4.3) on the numerical fluxes, there is a constant $C>0$ such that for every $\varphi \in C_{0}^{\infty}(M \times$ $\left.\mathbb{R}_{+}, \mathbb{R}_{+}\right)$and $\kappa \in\left[U_{m}, U_{M}\right]$ we have

$$
\begin{aligned}
\int_{0}^{T} & \int_{M}\left|u^{h}(x, t)-\kappa\right| \varphi_{t}(x, t) \mathrm{dv}_{g}(x) \mathrm{d} t+\int_{M}\left|u_{0}(x)-\kappa\right| \varphi(x, 0) \mathrm{dv}_{g}(x) \\
& +\int_{0}^{T} \int_{M}\left(f\left(x, u^{h}(x, t) \top \kappa\right)-f\left(x, u^{h}(x, t) \perp \kappa\right)\right) \cdot \nabla_{g} \varphi(x, t) \mathrm{d} v_{g}(x) \mathrm{d} t \\
\geq & -\int_{M}\left|u^{h}(x, 0)-u_{0}(x)\right| \varphi(x, 0) \mathrm{dv}_{g}(x) \\
& -2 \int_{0}^{T} \sum_{K \in \mathcal{T}} \sum_{e \in \partial K}|e|\left[\max _{(c, d) \in C\left(u_{K}^{h}, u_{K}^{h}\right)}\left|f_{K, e}(c, d)-f_{K, e}(c, c)\right|+C \delta(K)\right] r_{K, e}(t) \mathrm{d} t
\end{aligned}
$$

with

$$
r_{K, e}(t):=\frac{1}{|K||e|} \int_{e} \int_{K} \int_{0}^{d_{g}(x, y)}\left\|\nabla_{g} \varphi\left(\gamma_{x y}(\theta), t\right)\right\|_{g} \mathrm{~d} \theta \operatorname{dv}_{e}(y) \operatorname{dv}_{g}(x) .
$$

Proof. We start by using $\psi(t):=\frac{1}{|K|} \int_{K} \varphi(x, t) \mathrm{dv}_{g}(x)$ as test function in the weak discrete entropy inequality (Lem. 5.5) and summing over all $K \in \mathcal{T}$. Using that $f$ is geometry compatible (1.4) and the consistency property of the numerical fluxes (4.2) we get $T_{1}+T_{2} \leq 0$ with

$$
\begin{aligned}
T_{1}:= & -\int_{0}^{T} \sum_{K \in \mathcal{T}}\left|u_{K}^{h}(t)-\kappa\right|\left(\int_{K} \varphi(x, t) \mathrm{dv}_{g}(x)\right)_{t} \mathrm{~d} t-\sum_{K \in \mathcal{T}}\left|u_{K}^{h}(0)-\kappa\right| \int_{K} \varphi(x, 0) \mathrm{dv}_{g}(x) \\
= & -\int_{0}^{T} \int_{M}\left|u^{h}(x, t)-\kappa\right| \varphi_{t}(x, t) \mathrm{dv}_{g}(x) \mathrm{d} t-\int_{M}\left|u^{h}(x, 0)-\kappa\right| \varphi(x, 0) \mathrm{dv}_{g}(x) \\
T_{2}:= & \int_{0}^{T} \sum_{K \in \mathcal{T}} \sum_{e \in \partial K} \frac{|e|}{|K|}\left(f_{K, e}\left(u_{K}^{h}(t) \top \kappa, u_{K_{e}}^{h}(t) \top \kappa\right)\right. \\
& -f_{K, e}\left(u_{K}^{h}(t) \top \kappa, u_{K}^{h}(t) \top \kappa\right)-f_{K, e}\left(u_{K}^{h}(t) \perp \kappa, u_{K_{e}}^{h}(t) \perp \kappa\right) \\
& \left.+f_{K, e}\left(u_{K}^{h}(t) \perp \kappa, u_{K}^{h}(t) \perp \kappa\right)\right) \int_{K} \varphi(x, t) \mathrm{dv}_{g}(x) \mathrm{d} t .
\end{aligned}
$$

Now let

$$
\begin{aligned}
& T_{10}:=-\int_{0}^{T} \int_{M}\left|u^{h}(x, t)-\kappa\right| \varphi_{t}(x, t) \mathrm{dv}_{g}(x) \mathrm{d} t-\int_{M}\left|u_{0}(x)-\kappa\right| \varphi(x, 0) \mathrm{dv}_{g}(x) \\
& T_{20}:=-\int_{0}^{T} \int_{M}\left(f\left(x, u^{h}(x, t) \top \kappa\right)-f\left(x, u^{h}(x, t) \perp \kappa\right)\right) \nabla_{g} \varphi(x, t) \mathrm{dv}_{g}(x) \mathrm{d} t .
\end{aligned}
$$


We are going to estimate $\left|T_{1}-T_{10}\right|$ and $\left|T_{2}-T_{20}\right|$. Obviously we have

$$
\begin{aligned}
\left|T_{1}-T_{10}\right| & \leq \int_{M}|| u^{h}(x, 0)-\kappa|-| u_{0}(x)-\kappa|| \varphi(x, 0) \mathrm{dv}_{g}(x) \\
& \leq \int_{M}\left|u^{h}(x, 0)-u_{0}(x)\right| \varphi(x, 0) \mathrm{dv}_{g}(x) .
\end{aligned}
$$

Due to the geometry compatibility of the numerical fluxes (1.4) we have

$$
\begin{aligned}
T_{20}= & -\int_{0}^{T} \sum_{K \in \mathcal{T}} \int_{K} \nabla_{g} \cdot\left[\left(f\left(x, u_{K}^{h}(t) \top \kappa\right)-f\left(x, u_{K}^{h}(t) \perp \kappa\right)\right) \varphi(x, t)\right] \operatorname{dv}_{g}(x) \\
= & -\int_{0}^{T} \sum_{K \in \mathcal{T}} \sum_{e \in \partial K} \int_{e}\left(f\left(x, u_{K}^{h}(t) \top \kappa\right)-f\left(x, u_{K}^{h}(t) \perp \kappa\right)\right) n_{K, e}(x) \varphi(x, t) \mathrm{dv}_{e}(x) \\
& +\int_{0}^{T} \sum_{K \in \mathcal{T}} \sum_{e \in \partial K}|e|\left(f_{K, e}\left(u_{K}^{h}(t) \top \kappa, u_{K_{e}}^{h}(t) \top \kappa\right)\right. \\
& \left.-f_{K, e}\left(u_{K}^{h}(t) \perp \kappa, u_{K_{e}}^{h}(t) \perp \kappa\right)\right) \frac{1}{|e|} \int_{e} \varphi(x, t) \mathrm{dv}_{e}(x) \mathrm{d} t
\end{aligned}
$$

because the last summand is zero due to the fact that each face $e$ is a face of exactly two polyhedra and the conservation property (4.1) of the numerical fluxes. Therefore we get

$$
\begin{aligned}
\left|T_{2}-T_{20}\right| \leq & \int_{0}^{T} \mid \sum_{K \in \mathcal{T}} \sum_{e \in \partial K} \int_{e}\left(f\left(y, u_{K}^{h}(t) \top \kappa\right)-f\left(y, u_{K}^{h}(t) \perp \kappa\right)\right) \\
& \times n_{K, e}(y)\left(\varphi(y, t)-\frac{1}{|K|} \int_{K} \varphi(x, t) \mathrm{dv}_{g}(x)\right) \mathrm{dv}_{e}(y) \\
& +|e|\left(f_{K, e}\left(u_{K}^{h}(t) \top \kappa, u_{K_{e}}^{h}(t) \top \kappa\right)-f_{K, e}\left(u_{K}^{h}(t) \perp \kappa, u_{K_{e}}^{h}(t) \perp \kappa\right)\right) \\
& \times\left(\frac{1}{|K|} \int_{K} \varphi(x, t) \operatorname{dv}_{g}(x)-\frac{1}{|e|} \int_{e} \varphi(y, t) \operatorname{dv}_{e}(y)\right) \mid \mathrm{d} t .
\end{aligned}
$$

To estimate this further we need an estimate for

$$
\left|f\left(x, u_{K}^{h}(t) \top \kappa\right) \cdot n_{K, e}(x)-f_{K, e}\left(u_{K}^{h} \top \kappa, u_{K}^{h} \top \kappa\right)\right|
$$

for every $x \in e$. The fact that $f \cdot n_{K, e}$ is continuous with respect to the space variable implies due to (4.2)

$$
\begin{aligned}
f_{K, e}\left(u_{K}^{h} \top \kappa, u_{K}^{h} \top \kappa\right) & =\frac{1}{|e|} \int_{e} f\left(x, u_{K}^{h}(t) \top \kappa\right) n_{K, e}(x) \mathrm{dv}_{e}(x) \\
& =f\left(\xi, u_{K}^{h}(t) \top \kappa\right) n_{K, e}(\xi)
\end{aligned}
$$

for some $\xi \in e$. Due to the bound $\bar{R}$ for the principal curvatures of the faces we have

$$
\begin{aligned}
t\left\langle f\left(x,\left(u_{K}^{h}(t) \top \kappa\right)\right), n_{K, e}(x)\right\rangle_{g} & =\left\langle\nabla_{t} f\left(x,\left(u_{K}^{h}(t) \top \kappa\right)\right), n_{K, e}(x)\right\rangle_{g}+\left\langle f\left(x,\left(u_{K}^{h}(t) \top \kappa\right)\right), \nabla_{t} n_{K, e}(x)\right\rangle_{g} \\
& \leq C_{2}+\mathrm{d} \bar{R}\|f\|_{\infty} .
\end{aligned}
$$

Thus we have

$$
\begin{aligned}
\left|f\left(x,\left(u_{K}^{h}(t) \top \kappa\right)\right) n_{K, e}(x)-f_{K, e}\left(u_{K}^{h} \top \kappa, u_{K}^{h} \top \kappa\right)\right| & =\left|f\left(x,\left(u_{K}^{h}(t) \top \kappa\right)\right) n_{K, e}(x)-f\left(\xi,\left(u_{K}^{h}(t) \top \kappa\right)\right) n_{K, e}(\xi)\right| \\
& \leq \delta(e)\left(C_{2}+\mathrm{d} \bar{R}\|f\|_{\infty}\right) \leq \delta(K)\left(C_{2}+\mathrm{d} \bar{R}\|f\|_{\infty}\right) .
\end{aligned}
$$


Using a similar estimate for the $\perp$ case we get from (5.6)

$$
\begin{aligned}
\left|T_{2}-T_{20}\right| \leq & \int_{0}^{T} \sum_{K \in \mathcal{T}} \sum_{e \in \partial K}\left[|e| \mid-f_{K, e}\left(u_{K}^{h}(t) \top \kappa, u_{K}^{h}(t) \top \kappa\right)\right. \\
& +f_{K, e}\left(u_{K}^{h}(t) \perp \kappa, u_{K}^{h}(t) \perp \kappa\right) \\
& +f_{K, e}\left(u_{K}^{h}(t) \top \kappa, u_{K_{e}}^{h}(t) \top \kappa\right)-f_{K, e}\left(u_{K}^{h}(t) \perp \kappa, u_{K_{e}}^{h}(t) \perp \kappa\right) \mid \\
& \times \frac{1}{|e||K|} \int_{e} \int_{K}|\varphi(x, t)-\varphi(y, t)| \operatorname{dv}_{e}(y) \operatorname{dv}_{g}(x) \\
& \left.+\delta(K) C \int_{e}\left|\varphi(y, t)-\frac{1}{|K|} \int_{K} \varphi(x, t) \operatorname{dv}_{g}(x)\right| \operatorname{dv}_{e}(y)\right] .
\end{aligned}
$$

For $h$ small enough, $x \in K$ and $y \in e \in \partial K$ let $\gamma_{x y}$ denote the unique minimizing geodesic from $x$ to $y$ parametrized by arc length. Then we have

$$
\begin{aligned}
\frac{1}{|K||e|} \int_{e} \int_{K}|\varphi(x, t)-\varphi(y, t)| \mathrm{dv}_{e}(y) \mathrm{dv}_{g}(x) & =\frac{1}{|K||e|} \int_{e} \int_{K}\left|\int_{0}^{d_{g}(x, y)}\left\langle\nabla_{g} \varphi\left(\gamma_{x y}(s), t\right), \gamma_{x y}^{\prime}(s)\right\rangle_{g} \mathrm{~d} s\right| \mathrm{dv}_{g}(x) \mathrm{dv}_{e}(y) \\
& =\frac{1}{|K||e|} \int_{e} \int_{K} \int_{0}^{d_{g}(x, y)}\left\|\nabla_{g} \varphi\left(\gamma_{x y}(\theta), t\right)\right\|_{g} \mathrm{~d} \theta \mathrm{dv}_{e}(y) \mathrm{dv}_{g}(x)
\end{aligned}
$$

This finally yields

$$
\begin{aligned}
\left|T_{2}-T_{20}\right| \leq & \int_{0}^{T} \sum_{K \in \mathcal{T}} \sum_{e \in \partial K}|e|\left[\mid-f_{K, e}\left(u_{K}^{h}(t) \top \kappa, u_{K}^{h}(t) \top \kappa\right)\right. \\
& +f_{K, e}\left(u_{K}^{h}(t) \perp \kappa, u_{K}^{h}(t) \perp \kappa\right)+f_{K, e}\left(u_{K}^{h}(t) \top \kappa, u_{K_{e}}^{h}(t) \top \kappa\right) \\
& \left.-f_{K, e}\left(u_{K}^{h}(t) \perp \kappa, u_{K_{e}}^{h}(t) \perp \kappa\right) \mid+C \delta(K)\right] r_{K, e}
\end{aligned}
$$

with $r_{K, e}$ given in (5.5).

Now we want to estimate the right hand side of the above inequality (5.7). Due to the monotonicity (4.3) of the numerical fluxes we observe for $u_{K}^{h} \geq u_{K_{e}}^{h}$

$$
\begin{aligned}
0 & \leq-f_{K, e}\left(u_{K}^{h} \top \kappa, u_{K}^{h} \top \kappa\right)+f_{K, e}\left(u_{K}^{h} \top \kappa, u_{K_{e}}^{h} \top \kappa\right) \\
& \leq \max _{u_{K_{e}}^{h} \leq c \leq d \leq u_{K}^{h}}\left(-f_{K, e}(d, d)+f_{K, e}(d, c)\right) \\
& =\max _{(c, d) \in C\left(u_{K}^{h}, u_{K_{e}}^{h}\right)}\left|f_{K, e}(c, d)-f_{K, e}(c, c)\right|
\end{aligned}
$$

and for $u_{K}^{h} \leq u_{K_{e}}^{h}$

$$
\begin{aligned}
0 & \leq f_{K, e}\left(u_{K}^{h} \top \kappa, u_{K}^{h} \top \kappa\right)-f_{K, e}\left(u_{K}^{h} \top \kappa, u_{K_{e}}^{h} \top \kappa\right) \\
& \leq \max _{u_{K}^{h} \leq c \leq d \leq u_{K_{e}}^{h}}\left(+f_{K, e}(c, c)-f_{K, e}(c, d)\right) \\
& =\max _{(c, d) \in C\left(u_{K}^{h}, u_{K_{e}}^{h}\right)}\left|f_{K, e}(c, d)-f_{K, e}(c, c)\right| .
\end{aligned}
$$


There are similar estimates for $\perp$ instead of $\top$ which show that

$$
\begin{aligned}
\sum_{K \in \mathcal{T}} \sum_{e \in \partial K}|e|\left[\mid f_{K, e}\left(u_{K}^{h}(t) \top \kappa, u_{K}^{h}(t) \top \kappa\right)-f_{K, e}\left(u_{K}^{h}(t) \top \kappa, u_{K_{e}}^{h}(t) \top \kappa\right)\right. & \\
& \left.-f_{K, e}\left(u_{K}^{h}(t) \perp \kappa, u_{K}^{h}(t) \perp \kappa\right)+f_{K, e}\left(u_{K}^{h}(t) \perp \kappa, u_{K_{e}}^{h}(t) \perp \kappa\right) \mid+C \delta(K)\right] \\
\leq & 2 \sum_{K \in \mathcal{T}} \sum_{e \in \partial K}|e|\left[\max _{(c, d) \in C\left(u_{K}^{h}, u_{K_{e}}^{h}\right)}\left|f_{K, e}(c, d)-f_{K, e}(c, c)\right|+C \delta(K)\right]
\end{aligned}
$$

This implies together with (5.7)

$$
\left|T_{2}-T_{20}\right| \leq \int_{0}^{T} 2 \sum_{K \in \mathcal{T}} \sum_{e \in \partial K}|e|\left[\max _{(c, d) \in C\left(u_{K}^{h}, u_{K_{e}}^{h}\right)}\left|f_{K, e}(c, d)-f_{K, e}(c, c)\right|+C \delta(K)\right] r_{K, e}(t)
$$

which implies the lemma.

The next lemma is a very important step in the convergence proof. There will be different estimates for $d=1$ and $d \geq 2$. This is due to the fact that while we have the TVD property (Lem. 5.3) in the $d=1$ case, we only have the weak BV estimate (Lem. 5.4) in the $d \geq 2$ case. The proof will be done for $d \geq 2$ only. The proof for $d=1$ follows from the same arguments using Lemma 5.3 instead of Lemma 5.4.

Lemma 5.7. Provided the assumptions from Lemma 5.6 there exists a constant $C>0$ depending only on $M$, $g, u_{0},\left\{f_{K, e}\right\}, f, \beta, k, \bar{R}, N_{c}$ such that for small enough $h$ and every test function $\alpha \in C_{0}^{\infty}\left(M \times \mathbb{R}_{+}, \mathbb{R}_{+}\right)$the following inequality holds

$$
\begin{aligned}
\int_{M \times \mathbb{R}_{+}} \mid u^{h}(x, t) & -u(x, t) \mid \alpha_{t}(x, t) \mathrm{dv}_{g}(x) \mathrm{d} t \\
& +\int_{M \times \mathbb{R}_{+}}\left[f\left(x, u(x, t) \top u^{h}(x, t)\right)-f\left(x, u(x, t) \perp u^{h}(x, t)\right)\right] \cdot \nabla_{g} \alpha(x, t) \mathrm{dv}_{g}(x) \mathrm{d} t \\
\geq & \left\{\begin{array}{l}
-C h^{\frac{1}{2}}: d=1 \\
-C h^{\frac{1}{4}}: d \geq 2 .
\end{array}\right.
\end{aligned}
$$

Proof. The proof is based on a doubling of variables argument. We recall the entropy inequality (1.3) fulfilled by the entropy solution $u$ of $(1.1),(1.2)$

$$
\begin{aligned}
& \int_{M \times \mathbb{R}_{+}}|u(y, s)-\kappa| \varphi_{s}(y, s)+[f(y, u(y, s) \top \kappa)-f(y, u(y, s) \perp \kappa)] \cdot \nabla_{g} \varphi(y, s) \operatorname{dv}_{g}(y) \mathrm{d} s \\
&+\int_{M}\left|u_{0}(y)-\kappa\right| \varphi(y, 0) \operatorname{dv}_{g}(y) \geq 0
\end{aligned}
$$

for all $\kappa \in \mathbb{R}$ and $\varphi \in C_{0}^{\infty}\left(M \times \mathbb{R}_{+}, \mathbb{R}_{+}\right)$. In (1.3) we set $\kappa=u^{h}(x, t)$ and $\varphi(y, s)=\alpha(x, t) \chi_{\varepsilon}(x, y) \psi_{\varepsilon}(t-s)$, where $\chi_{\varepsilon}$ and $\psi_{\varepsilon}$ are cut-off functions as defined in Section 2.3. Now we integrate this equation with respect to $x$ and $t$. In the continuous entropy inequality from Lemma 5.6 we set $\kappa=u(y, s)$ and $\varphi(x, t)=\alpha(x, t) \chi_{\varepsilon}(x, y) \psi_{\varepsilon}(t-s)$ 
and integrate with respect to $y$ and $s$. Adding both equations yields

$$
\begin{aligned}
\int_{M^{2} \times \mathbb{R}_{+}^{2}} \mid u^{h}(x, t)-u( & , s) \mid \alpha_{t}(x, t) \chi_{\varepsilon}(x, y) \psi_{\varepsilon}(t-s) \mathrm{dv}_{g}(x) \mathrm{dv}_{g}(y) \mathrm{d} t \mathrm{~d} s \\
& +\int_{M^{2} \times \mathbb{R}_{+}^{2}}\left[f\left(y, u(y, s) \top u^{h}(x, t)\right)-f\left(y, u(y, s) \perp u^{h}(x, t)\right)\right] \\
& \times \alpha(x, t) \nabla_{g, y} \chi_{\varepsilon}(x, y) \psi_{\varepsilon}(t-s) \mathrm{dv}_{g}(x) \mathrm{dv}_{g}(y) \mathrm{d} t \mathrm{~d} s \\
& +\int_{M^{2} \times \mathbb{R}_{+}^{2}}\left[f\left(x, u(y, s) \top u^{h}(x, t)\right)-f\left(x, u(y, s) \perp u^{h}(x, t)\right)\right] \\
& \times \nabla_{g} \alpha(x, t) \chi_{\varepsilon}(x, y) \psi_{\varepsilon}(t-s) \mathrm{dv}_{g}(x) \mathrm{dv}_{g}(y) \mathrm{d} t \mathrm{~d} s \\
& +\int_{M^{2} \times \mathbb{R}_{+}^{2}}\left[f\left(x, u(y, s) \top u^{h}(x, t)\right)-f\left(x, u(y, s) \perp u^{h}(x, t)\right)\right] \\
& \times \alpha(x, t) \nabla_{g, x} \chi_{\varepsilon}(x, y) \psi_{\varepsilon}(t-s) \mathrm{dv}_{g}(x) \mathrm{dv}_{g}(y) \mathrm{d} t \mathrm{~d} s \\
& +\int_{M^{2} \times \mathbb{R}_{+}}\left|u_{0}(x)-u(y, s)\right| \alpha(x, 0) \chi_{\varepsilon}(x, y) \psi_{\varepsilon}(-s) \mathrm{d} \mathrm{v}_{g}(x) \mathrm{dv} \mathrm{v}_{g}(y) \mathrm{d} s \\
\geq & -\int_{M^{2} \times \mathbb{R}_{+}}\left|u^{h}(x, 0)-u_{0}(x)\right| \alpha(x, 0) \chi_{\varepsilon}(x, y) \psi_{\varepsilon}(-s) \mathrm{d} s \mathrm{dv}_{g}(y) \mathrm{d} \mathrm{v}_{g}(x) \\
& -2 \int_{M \times \mathbb{R}_{+}} \int_{0}^{T} \sum_{K \in \mathcal{T}} \sum_{e \in \partial K}|e|\left[\max _{(c, d) \in C\left(u_{K}^{h}, u_{K_{e}}^{h}\right)}\left|f_{K, e}(c, d)-f_{K, e}(c, c)\right|\right. \\
& +C \delta(K)] r_{K, e}(t) \mathrm{d} t \mathrm{~d} s \mathrm{dv}_{g}(y) .
\end{aligned}
$$

We will start with the most difficult summand. Let $E_{2}$ be the sum of the second and fourth summand, i.e.

$$
\begin{aligned}
E_{2}:= & \int_{M^{2} \times \mathbb{R}_{+}^{2}}\left\{\left[f\left(y, u(y, s) \top u^{h}(x, t)\right)-f\left(y, u(y, s) \perp u^{h}(x, t)\right)\right] \cdot \nabla_{g, y} \chi_{\varepsilon}(x, y)\right. \\
& \left.+\left[f\left(x, u(y, s) \top u^{h}(x, t)\right)-f\left(x, u(y, s) \perp u^{h}(x, t)\right)\right] \cdot \nabla_{g, x} \chi_{\varepsilon}(x, y)\right\} \\
& \times \alpha(x, t) \psi_{\varepsilon}(t-s) \operatorname{dv}_{g}(x) \operatorname{dv}_{g}(y) \mathrm{d} t \mathrm{~d} s .
\end{aligned}
$$

We also define

$$
\begin{aligned}
E_{2 b}:= & \int_{M^{2} \times \mathbb{R}_{+}^{2}}\left\{\left[f\left(y, u(x, t) \top u^{h}(x, t)\right)-f\left(y, u(x, t) \perp u^{h}(x, t)\right)\right] \cdot \nabla_{g, y} \chi_{\varepsilon}(x, y)\right. \\
& \left.+\left[f\left(x, u(x, t) \top u^{h}(x, t)\right)-f\left(x, u(x, t) \perp u^{h}(x, t)\right)\right] \cdot \nabla_{g, x} \chi_{\varepsilon}(x, y)\right\} \alpha(x, t) \psi_{\varepsilon}(t-s) \mathrm{dv}_{g}(x) \mathrm{dv}_{g}(y) \mathrm{d} t \mathrm{~d} s .
\end{aligned}
$$

For ease of notation we will from now on omit the $\perp$-terms for the estimate for $E_{2}$. Adding zero we get

$$
\begin{aligned}
E_{2 b} \stackrel{(2.3),(2.9)}{=} & \int_{M^{2} \times \mathbb{R}_{+}^{2}} f\left(y, u(x, t) \top u^{h}(x, t)\right) \alpha(x, t) \nabla_{g, y} \chi_{\varepsilon}(x, y) \psi_{\varepsilon}(t-s) \\
& -\left(T \exp _{x}\right)_{\exp _{x}^{-1}(y)}\left(f\left(x, u(x, t) \top u^{h}(x, t)\right)\right) \alpha(x, t) \nabla_{g, y} \chi_{\varepsilon}(x, y) \psi_{\varepsilon}(t-s) \\
& +\left(\left(T \exp _{x}\right)_{\exp _{x}^{-1}(y)}\left(f\left(x, u(x, t) \top u^{h}(x, t)\right)\right)-P_{x y}\left(f\left(x, u(x, t) \top u^{h}(x, t)\right)\right)\right) \\
& \times \nabla_{g, y} \chi_{\varepsilon}(x, y) \alpha(x, t) \psi_{\varepsilon}(t-s) \operatorname{dv}_{g}(x) \operatorname{dv}_{g}(y) \mathrm{d} t \mathrm{~d} s .
\end{aligned}
$$


Now using integration by parts w.r.t. $y$ the first summand vanishes because $f(\cdot, s)$ is divergence free for fixed $s \in \mathbb{R}(1.4)$, the absolute value of the second summand is smaller or equal $C \varepsilon$ which can be seen after integration by parts w.r.t. $y$ by (2.10). To get an estimate for the third summand, denoted $I_{3}$, we observe that for every $x \in M$ and $\varepsilon<2 R$

$$
\int_{B_{\varepsilon}(x)} \operatorname{dv}_{g}(y)=\int_{B_{\varepsilon}(0)}\left|\operatorname{det}\left(T \exp _{x}\right)_{v}\right| \mathrm{d} v<C \sup _{x \in M,\|v\|_{g}<\varepsilon}\left|\operatorname{det}\left(T \exp _{x}\right)_{v}\right| \varepsilon^{d} .
$$

This yields due to $(2.7)$ and $(2.12)$

$$
\begin{aligned}
\left|I_{3}\right| & \leq \int_{M^{2} \times \mathbb{R}_{+}^{2}} C \mathbb{I}_{\left\{d_{g}(x, y)<\varepsilon\right\}} \frac{\|f\|_{\infty}}{\varepsilon^{d-1}} \alpha(x, t) \psi_{\varepsilon}(t-s) \operatorname{dv}_{g}(x) \mathrm{dv}_{g}(y) \mathrm{d} t \mathrm{~d} s \\
& \leq \int_{M \times \mathbb{R}_{+}} C \sup _{x \in M,\|v\|_{g}<\varepsilon}\left|\operatorname{det}\left(T \exp _{x}\right)_{v}\right| \varepsilon \alpha(x, t) \mathrm{dv}_{g}(x) \mathrm{d} t \\
& \leq C \varepsilon .
\end{aligned}
$$

So finally $\left|E_{2 b}\right|<C \varepsilon$, and it remains to show $\left|E_{2}-E_{2 b}\right|<C \varepsilon$. Introducing the following notation omitting the dependence on $t$ and $s$ :

$$
\begin{aligned}
h(x, y, \tau) & :=f\left(\gamma_{x y}(\tau), u(y, s) \top u^{h}(x, t)\right) \cdot \gamma_{x y}^{\prime}(\tau), \\
\tilde{h}(x, y, \tau) & :=f\left(\gamma_{x y}(\tau), u(x, t) \top u^{h}(x, t)\right) \cdot \gamma_{x y}^{\prime}(\tau), \\
D(x, y) & :=h\left(x, y, d_{g}(x, y)\right)-h(x, y, 0)-\tilde{h}\left(x, y, d_{g}(x, y)\right)+\tilde{h}(x, y, 0),
\end{aligned}
$$

we have due to $(2.2)$ and the definition of $\chi_{\varepsilon}$ in $(2.5)$

$$
\begin{aligned}
\mid E_{2}- & E_{2 b}|\leq| \int_{M^{2} \times \mathbb{R}_{+}^{2}} D(x, y) \alpha(x, t) \frac{1}{\varepsilon^{d+1}} \chi^{\prime}\left(\frac{d_{g}(x, y)}{\varepsilon}\right) \psi_{\varepsilon}(t-s) \mathrm{dv}_{g}(x) \mathrm{dv}_{g}(y) \mathrm{d} t \mathrm{~d} s \mid \\
|D| & \leq\left|\int_{0}^{d_{g}(x, y)} \frac{\mathrm{d}}{\mathrm{d} \tau}(h(x, y, \tau)-\tilde{h}(x, y, \tau)) \mathrm{d} \tau\right| \\
& =\left|\int_{0}^{d_{g}(x, y)}\left\langle\nabla_{\gamma_{x y}^{\prime}}\left(f\left(\cdot, u(y, s) \top u^{h}(x, t)\right)-f\left(\cdot, u(x, t) \top u^{h}(x, t)\right)\right), \gamma_{x y}^{\prime}\right\rangle \mathrm{d} \tau\right| \\
& \stackrel{(1.4)}{=} \leq C|u(y, s)-u(x, t)| d_{g}(x, y) .
\end{aligned}
$$

Inserting this in (5.10) and using the boundedness of $\chi^{\prime}$ we have

$$
\left|E_{2}-E_{2 b}\right| \leq C \int_{M^{2} \times \mathbb{R}_{+}^{2}}|u(y, s)-u(x, t)||\alpha(x, t)| \frac{1}{\varepsilon^{d}} \mathbb{I}_{\left\{d_{g}(x, y)<\varepsilon\right\}} \psi_{\varepsilon}(t-s) \operatorname{dv}_{g}(x) \operatorname{dv}_{g}(y) \mathrm{d} t \mathrm{~d} s .
$$

Now we cover $M$ with finitely many geodesic balls $B_{r}\left(x_{1}\right), \ldots, B_{r}\left(x_{N}\right)$, where $r<R$ and $R$ is the constant from Lemma 2.2. We furthermore restrict to the $\varepsilon<r$ case. Because the derivative of $\exp _{x_{i}}^{-1}$ is bounded there exists a constant $C_{i}>0$ such that

$$
d_{g}\left(\exp _{x_{i}}(a), \exp _{x_{i}}(b)\right)>C_{i}\|a-b\|_{g}, \forall a \in B_{r}(0), b \in B_{2 r}(0)
$$

which implies

$$
\mathbb{I}_{\left\{d_{g}\left(\exp _{x_{i}}(a), \exp _{x_{i}}(b)\right)<\varepsilon\right\}} \leq \mathbb{I}_{\left\{\|b-a\|_{g}<\frac{\varepsilon}{C_{i}}\right\}} .
$$


Hence we have by

$$
\begin{aligned}
\left|E_{2}-E_{2 b}\right| \leq & C \sum_{i} \int_{\mathbb{R}_{+}^{2}} \int_{B_{r}(0)} \int_{B_{2 r}(0)}\left|u\left(\exp _{x_{i}}(a), t\right)-u\left(\exp _{x_{i}}(b), s\right)\right| \\
& \times \varepsilon^{-d_{1}} \mathbb{I}_{\left\{\|b-a\|_{g}<\frac{\varepsilon}{C_{i}}\right\}} \mathbb{I}_{\operatorname{supp} \alpha} \psi_{\varepsilon}(t-s) \mathrm{d} a \mathrm{~d} b \mathrm{~d} t \mathrm{~d} s \\
\leq & C \varepsilon
\end{aligned}
$$

because each $u \circ \exp _{x_{i}}$ has bounded variation. Finally we have

$$
\left|E_{2}\right| \leq C \varepsilon
$$

Let

$$
\begin{aligned}
E_{1} & :=\int_{M^{2} \times \mathbb{R}_{+}^{2}}\left|u^{h}(x, t)-u(y, s)\right| \alpha_{t}(x, t) \chi_{\varepsilon}(x, y) \psi_{\varepsilon}(t-s) \operatorname{dv}_{g}(x) \mathrm{dv}_{g}(y) \mathrm{d} t \mathrm{~d} s \\
E_{1 b} & :=\int_{M \times \mathbb{R}_{+}}\left|u^{h}(x, t)-u(x, t)\right| \alpha_{t}(x, t) \operatorname{dv}_{g}(x) \mathrm{d} t .
\end{aligned}
$$

Due to (2.8) we have

$$
\left|E_{1}-E_{1 b}\right| \leq \int_{M^{2} \times \mathbb{R}_{+}^{2}}|u(x, t)-u(y, s)|\left|\alpha_{t}(x, t)\right| \chi_{\varepsilon}(x, y) \psi_{\varepsilon}(t-s) \mathrm{dv}_{g}(x) \mathrm{dv}_{g}(y) \mathrm{d} t \mathrm{~d} s+C \varepsilon .
$$

To estimate the first part of the right hand side we again cover $M$ with balls $B_{r}\left(x_{i}\right)$ like in the estimate for $E_{2}$. From the definition of $\chi_{\varepsilon}$ in (2.5) we know that $\chi$ is nonincreasing for positive $x$, this yields the following inequality

$$
\begin{aligned}
\left|E_{1}-E_{1 b}\right| \leq & \sum_{i} \int_{\mathbb{R}_{+}^{2}} \int_{B_{r_{i}}(0)} \int_{B_{r_{i}}(0)}\left|u\left(\exp _{x_{i}}(a), t\right)-u\left(\exp _{x_{i}}(b), s\right)\right| \\
& \times\left|\alpha_{t}\left(\exp _{x_{i}}(a), t\right)\right| \frac{1}{\varepsilon^{d}} \chi\left(\frac{C_{i}\|b-a\|_{g}}{\varepsilon}\right) \psi_{\varepsilon}(t-s) \mathrm{d} a \mathrm{~d} b \mathrm{~d} t \mathrm{~d} s+C \varepsilon \\
\leq & C \varepsilon
\end{aligned}
$$

because the $L^{1}$-norms of the $\frac{1}{\varepsilon^{d}} \chi\left(\frac{C_{i}\|b-a\|_{g}}{\varepsilon}\right)$ are uniformly bounded with respect to $\varepsilon$. The constants $C_{i}$ were chosen like in (5.11).

Let

$$
\begin{aligned}
E_{3}:= & \int_{M^{2} \times \mathbb{R}_{+}^{2}}\left[f\left(x, u(y, s) \top u^{h}(x, t)\right)-f\left(x, u(y, s) \perp u^{h}(x, t)\right)\right] \\
& \times \nabla_{g} \alpha(x, t) \chi_{\varepsilon}(x, y) \psi_{\varepsilon}(t-s) \mathrm{dv}_{g}(x) \mathrm{dv}_{g}(y) \mathrm{d} t \mathrm{~d} s \\
E_{3 b}:= & \int_{M \times \mathbb{R}_{+}}\left[f\left(x, u(x, t) \top u^{h}(x, t)\right)-f\left(x, u(x, t) \perp u^{h}(x, t)\right)\right] \cdot \nabla_{g} \alpha(x, t) \mathrm{dv}_{g}(x) \mathrm{d} t .
\end{aligned}
$$

Then we have

$$
\begin{aligned}
\left|E_{3}-E_{3 b}\right| & \leq C \int_{M^{2} \times \mathbb{R}_{+}^{2}}|u(y, s)-u(x, t)|\left\|\nabla_{g} \alpha(x, t)\right\|_{g} \chi_{\varepsilon}(x, y) \psi_{\varepsilon}(t-s) \mathrm{dv}_{g}(x) \mathrm{d} v_{g}(y) \mathrm{d} t \mathrm{~d} s+C \varepsilon \\
& \leq C \varepsilon
\end{aligned}
$$


like in the estimate for $E_{1}$. To estimate the fifth summand on the left hand side of (5.8), denoted $E_{4}$, we consider the entropy inequality (1.3) fulfilled by $u$. For fixed $x \in M$ we define

$$
\varphi(x, y, s):=\alpha(x, 0) \chi_{\varepsilon}(x, y) \int_{s}^{\infty} \psi_{\varepsilon}(-\tau) \mathrm{d} \tau
$$

and $\kappa=u_{0}(x)$. Then integration with respect to $x$ yields

$$
\begin{aligned}
& -\int_{M^{2} \times \mathbb{R}_{+}}\left|u(y, s)-u_{0}(x)\right| \alpha(x, 0) \chi_{\varepsilon}(x, y) \psi_{\varepsilon}(-s) \mathrm{dv}_{g}(x) \mathrm{dv}_{g}(y) \mathrm{d} s \\
& +\int_{M^{2} \times \mathbb{R}_{+}}\left(f\left(y, u(y, s) \top u_{0}(x)\right)-f\left(y, u(y, s) \perp u_{0}(x)\right)\right) \cdot \nabla_{g, y} \chi_{\varepsilon}(x, y) \alpha(x, 0)\left(\int_{s}^{\infty} \psi_{\varepsilon}(-\tau) \mathrm{d} \tau\right) \mathrm{dv}_{g}(x) \mathrm{dv}_{g}(y) \mathrm{d} s \\
& +\int_{M^{2}}\left|u_{0}(y)-u_{0}(x)\right| \alpha(x, 0) \chi_{\varepsilon}(x, y)\left(\int_{0}^{\infty} \psi_{\varepsilon}(-\tau) \mathrm{d} \tau\right) \mathrm{dv}_{g}(x) \mathrm{dv}_{g}(y) \geq 0
\end{aligned}
$$

We note that the first summand here is exactly $-E_{4}$, thus we denote the summands by $-E_{4}, E_{5}, E_{6}$ respectively. To estimate $E_{5}$ we define $E_{5 b}$ by

$$
E_{5 b}:=\int_{M^{2} \times \mathbb{R}_{+}} \int_{s}^{\infty}\left(f\left(y, u(y, s) \top u_{0}(y)\right)-f\left(y, u(y, s) \perp u_{0}(y)\right)\right) \cdot \nabla_{g, y} \chi_{\varepsilon}(x, y) \alpha(x, 0) \psi_{\varepsilon}(-\tau) \mathrm{d} \tau \mathrm{d} s \mathrm{dv}_{g}(x) \mathrm{dv}_{g}(y) .
$$

From now on we will omit the $\perp$-terms for convenience again, they are estimated in exactly the same way as the $T$-terms. Using integration by parts we have by $(2.3)$

$$
\begin{aligned}
& E_{5 b}=-\int_{M^{2} \times \mathbb{R}_{+}} \int_{s}^{\infty} P_{y x}\left(f\left(y, u(y, s) \top u_{0}(y)\right)\right) \cdot \nabla_{g, x} \chi_{\varepsilon}(x, y) \alpha(x, 0) \psi_{\varepsilon}(-\tau) \mathrm{d} \tau \mathrm{d} s \operatorname{dv}_{g}(x) \operatorname{dv}_{g}(y) \\
& =\int_{M^{2} \times \mathbb{R}_{+}} \int_{s}^{\infty}\left(\left(T \exp _{y}\right)_{\exp _{y}^{-1}(x)}\left(f\left(y, u(y, s) \top u_{0}(y)\right)\right)-P_{y x}\left(f\left(y, u(y, s) \top u_{0}(y)\right)\right)\right) \\
& \times \nabla_{g, x} \chi_{\varepsilon}(x, y) \alpha(x, 0) \psi_{\varepsilon}(-\tau) \mathrm{d} \tau \mathrm{d} s \mathrm{dv}_{g}(x) \mathrm{dv}_{g}(y) \\
& -\int_{M^{2} \times \mathbb{R}_{+}} \int_{s}^{\infty}\left(T \exp _{y}\right)_{\exp _{y}^{-1}(x)}\left(f\left(y, u(y, s) \top u_{0}(y)\right)\right) \cdot \nabla_{g, x} \chi_{\varepsilon}(x, y) \alpha(x, 0) \psi_{\varepsilon}(-\tau) \mathrm{d} \tau \mathrm{d} s \operatorname{dv}_{g}(x) \operatorname{dv}_{g}(y) \text {. }
\end{aligned}
$$

Now we get using (2.12) for the first and integration by parts for the second summand

$$
\begin{aligned}
\left|E_{5 b}\right| \leq & \left|\int_{M^{2} \times \mathbb{R}_{+}} \int_{s}^{\infty} C \mathbb{I}_{\left\{d_{g}(x, y)<\varepsilon\right\}} \frac{1}{\varepsilon^{d-1}}\|f\|_{\infty} \alpha(x, 0) \psi_{\varepsilon}(-\tau) \mathrm{d} \tau \mathrm{d} s \operatorname{dv}_{g}(x) \mathrm{dv}_{g}(y)\right| \\
& +\mid \int_{M^{2} \times \mathbb{R}_{+}} \int_{s}^{\infty} \operatorname{div}_{g, x}\left(T \exp _{y}\right)_{\exp _{y}^{-1}(x)}\left(f\left(y, u(y, s) \top u_{0}(y)\right)\right) \\
& \times \chi_{\varepsilon}(x, y) \alpha(x, 0) \psi_{\varepsilon}(-\tau) \mathrm{d} \tau \mathrm{d} s \operatorname{dv}_{g}(x) \operatorname{dv}_{g}(y) \mid \\
& +\mid \int_{M^{2} \times \mathbb{R}_{+}} \int_{s}^{\infty}\left(T \exp _{y}\right)_{\exp _{y}^{-1}(x)}\left(f\left(y, u(y, s) \top u_{0}(y)\right)\right) \\
& \times \nabla_{g} \alpha(x, 0) \chi_{\varepsilon}(x, y) \psi_{\varepsilon}(-\tau) \mathrm{d} \tau \mathrm{d} s \operatorname{dv}_{g}(x) \operatorname{dv}_{g}(y) \mid
\end{aligned}
$$

The first summand is smaller than $C \varepsilon$ because of (5.9). The second summand is smaller than $C \varepsilon$ due to (2.10) and the third summand has this property because, due to (2.11), the integrand is bounded and the support 
with respect to $s$ lies in $[0, \varepsilon]$. Finally we have $\left|E_{5 b}\right| \leq C \varepsilon$. Furthermore

$$
\left|E_{5}-E_{5 b}\right| \leq C \int_{M^{2} \times \mathbb{R}_{+}} \int_{s}^{\infty}\left|u_{0}(x)-u_{0}(y)\right|\left\|\nabla_{g, y} \chi_{\varepsilon}(x, y)\right\|_{g} \psi_{\varepsilon}(-\tau) \mathrm{d} \tau \mathrm{d} s \operatorname{dv}_{g}(y) \operatorname{dv}_{g}(x) .
$$

Integrating with respect to $\tau$ and $s$ yields

$$
\left|E_{5}-E_{5 b}\right| \leq C \int_{M^{2}}\left|u_{0}(x)-u_{0}(y)\right|\left\|\nabla_{g, y} \chi_{\varepsilon}(x, y)\right\|_{g} \varepsilon \operatorname{dv}_{g}(y) \operatorname{dv}_{g}(x)
$$

because the integral over $\tau$ is bounded by 1 and the support with respect to $s$ lies in $[0, \varepsilon]$. Then we use the fact

$$
\varepsilon\left\|\nabla_{g, y} \chi_{\varepsilon}(x, y)\right\|_{g} \leq C \varepsilon^{-d^{d}} \mathbb{I}_{\left\{d_{g}(x, y)<\varepsilon\right\}} .
$$

We cover $M$ with balls like in the estimate for $E_{2}$ again and a similar argument yields

$$
\left|E_{5}-E_{5 b}\right| \leq C \varepsilon .
$$

Another version of this argument implies

$$
\left|E_{6}\right| \leq C \varepsilon
$$

So we finally have

$$
\left|E_{4}\right| \leq C \varepsilon
$$

Now we have to find an estimate for the right hand side of (5.8): Keeping in mind the weak BV-estimate (5.4), the essential part of this estimate is an estimate for

$$
\left|\int_{M \times \mathbb{R}_{+}} r_{K, e}(t) \mathrm{d} s \mathrm{dv}_{g}(y)\right|,
$$

where $r_{K, e}$ was defined in (5.5). Because the test function $\varphi$ in the definition of $r_{K, e}$ now has the form

$$
\varphi(x, t)=\alpha(x, t) \chi_{\varepsilon}(x, y) \psi_{\varepsilon}(t-s)
$$

we have

$$
\begin{aligned}
\left|\int_{M \times \mathbb{R}_{+}} r_{K, e}(t) \mathrm{dv}_{g}(x) \mathrm{d} s\right|= & \frac{C}{|K||e|} \int_{M} \int_{\mathbb{R}_{+}} \int_{K} \int_{e} \int_{0}^{d_{g}(x, z)}\left\|\nabla_{g} \alpha\left(\gamma_{x z}(\theta), t\right)\right\|_{g} \\
& \times \chi_{\varepsilon}\left(\gamma_{x z}(\theta), y\right) \psi_{\varepsilon}(t-s) \mathrm{d} \theta \operatorname{dv}_{e}(z) \mathrm{dv}_{g}(x) \mathrm{d} s \operatorname{dv}_{g}(y) \\
& +\frac{C}{|K||e|} \int_{M} \int_{\mathbb{R}_{+}} \int_{K} \int_{e} \int_{0}^{d_{g}(x, z)}\left\|\nabla_{1} \chi_{\varepsilon}\left(\gamma_{x z}(\theta), y\right)\right\|_{g} \\
& \times \alpha\left(\gamma_{x z}(\theta), t\right) \psi_{\varepsilon}(t-s) \mathrm{d} \theta \operatorname{dv}_{e}(z) \operatorname{dv}_{g}(x) \mathrm{d} s \operatorname{dv}_{g}(y) .
\end{aligned}
$$

Now integration over $s, y$ yields that the first summand in (5.12) can be estimated by

$$
\frac{C}{|K \| e|} \int_{K} \int_{e} \int_{0}^{d_{g}(x, z)} \underbrace{\left\|\nabla_{g} \alpha\left(\gamma_{x z}(\theta), t\right)\right\|_{g}}_{\leq\left\|\nabla_{g} \alpha\right\|_{L^{\infty}(M)}}(1+C \varepsilon) \mathrm{d} \theta \operatorname{dv}_{e}(z) \operatorname{dv}_{g}(x) \leq C(1+\varepsilon) \delta(K) .
$$

To estimate the second summand in (5.12) we observe

$$
\left\|\nabla_{1} \chi_{\varepsilon}\left(\gamma_{x z}(\theta), y\right)\right\|_{g} \leq C \varepsilon^{-d-1} \mathbb{I}_{\left\{d_{g}\left(\gamma_{x z}(\theta), y\right) \leq \varepsilon\right\}} .
$$


Due to (5.9) integration over $s$ and $y$ yields that the second summand in (5.12) is smaller than

$$
\frac{C}{|K||e|} \int_{K} \int_{e} \int_{0}^{d_{g}(x, y)} C \varepsilon^{-1} \mathrm{~d} \theta \operatorname{dv}_{e}(z) \operatorname{dv}_{g}(x) \leq C \frac{\delta(K)}{\varepsilon} .
$$

So we have due to the weak BV estimate Lemma 5.4

$$
\begin{aligned}
\int_{M \times \mathbb{R}_{+}} \int_{0}^{T} \sum_{K \in \mathcal{T}} \sum_{e \in \partial K}\left[\max _{(c, d) \in C\left(u_{K}^{h}, u_{K_{e}}^{h}\right)}\left|e \| f_{K, e}(c, d)-f_{K, e}(c, c)\right|\right] r_{K, e} \mathrm{dv} g(x) \mathrm{d} t \mathrm{~d} s & \leq \frac{C}{\sqrt{h}}\left(\frac{h}{\varepsilon}+h+h \varepsilon\right) \\
& =C \sqrt{h}\left(\frac{1}{\varepsilon}+1+\varepsilon\right) .
\end{aligned}
$$

We observe that due to (3.1)-(3.4) we have

$$
\int_{0}^{T} \sum_{K \in \mathcal{T}} \sum_{e \in \partial K} C \delta(K)|e| \leq 3 C \beta^{-1} k
$$

Now it remains to estimate

$$
\int_{M^{2} \times \mathbb{R}_{+}}\left|u^{h}(x, 0)-u_{0}(x)\right| \alpha(x, 0) \chi_{\varepsilon}(x, y) \psi_{\varepsilon}(-s) \mathrm{d} s \operatorname{dv}_{g}(y) \operatorname{dv}_{g}(x) .
$$

Integrating with respect to $y, s$ yields that this term is smaller than

$$
C(1+C \varepsilon) \int_{M}\left|u^{h}(x, 0)-u_{0}(x)\right| \operatorname{dv}_{g}(x) \leq C(1+\varepsilon) h
$$

for $\varepsilon, h$ small enough by Lemma 3.2. This finally implies

$$
\begin{aligned}
\int_{M \times \mathbb{R}_{+}}\left|u^{h}(x, t)-u(x, t)\right| \alpha_{t}(x, t) \mathrm{dv}_{g}(x) \mathrm{d} t & \\
& +\int_{M \times \mathbb{R}_{+}}\left[f\left(x, u(x, t) \top u^{h}(x, t)\right)-f\left(x, u(x, t) \perp u^{h}(x, t)\right)\right] \cdot \nabla_{g} \alpha(x, t) \mathrm{dv}_{g}(x) \mathrm{d} t \\
\geq & -C\left(\varepsilon+C h+h \varepsilon+\frac{\sqrt{h}}{\varepsilon}+\sqrt{h}+2 \sqrt{h} \varepsilon+h \varepsilon\right) \\
= & -C\left(h^{\frac{1}{4}}+h+h^{\frac{3}{2}}+h^{\frac{1}{4}}+h^{\frac{1}{2}}+h+h^{\frac{3}{2}}\right)
\end{aligned}
$$

where we set $\varepsilon=h^{\frac{1}{4}}$ for the last equality.

Now we can state the main theorem which specifies the convergence rate. This result is more general with respect to the grids than the work of Amorim et al. [1] because they impose the lines joining the vertices of the polyhedra to be geodesic. Nevertheless we believe the techniques of their proof also work for the more general grids considered here.

Now the convergence proof is quite easy and only consists of choosing a sensible test function $\alpha$ in Lemma 5.7.

Theorem 5.8. Provided the assumptions of Lemma 5.7 hold, then we have for every time $T>0$ a constant $C>0$ depending only on $f, u_{0}, M, g, T,\left\{f_{K, e}\right\}, \beta, k, N_{c}, \bar{R}$ such that

$$
\int_{0}^{T} \int_{M}\left|u^{h}(x, t)-u(x, t)\right| \mathrm{dv}_{g}(x) \mathrm{d} t \leq\left\{\begin{array}{l}
C h^{\frac{1}{2}}: d=1 \\
C h^{\frac{1}{4}}: d \geq 2 .
\end{array}\right.
$$


Proof. For $t \geq 0$ we define

$$
\rho(t):= \begin{cases}(1-t) \exp \left(\frac{1}{t^{2}-1}\right) & : t \leq 1 \\ 0 & : t \geq 1\end{cases}
$$

and $\rho_{T}(t):=\rho\left(\frac{t}{2 T}\right)$. We have $\rho_{T}(t) \in[0, e]$ and there exists $\varepsilon>0$ such that $\rho^{\prime}(t)<-\varepsilon \forall t \in\left[0, \frac{1}{2}\right]$ and therefore $\rho_{T}^{\prime}(t)<\frac{-\varepsilon}{2 T} \forall t \in[0, T]$. Now we define $\alpha(x, t)=\rho_{T}(t)$, then we have $\alpha \in C_{0}^{\infty}\left(M \times \mathbb{R}_{+}, \mathbb{R}_{+}\right)$and $\nabla_{g} \alpha=0$ which implies

$$
\begin{aligned}
\frac{-\varepsilon}{2 T} \int_{M} \int_{0}^{T}\left|u^{h}(x, t)-u(x, t)\right| \mathrm{dv}_{g}(x) \mathrm{d} t & \geq \int_{M} \int_{0}^{T} \alpha_{t}(x, t)\left|u^{h}(x, t)-u(x, t)\right| \mathrm{dv}_{g}(x) \mathrm{d} t \\
& \geq\left\{\begin{array}{l}
-C h^{\frac{1}{2}}: d=1 \\
-C h^{\frac{1}{4}}: d \geq 2,
\end{array}\right.
\end{aligned}
$$

which proves the theorem.

\section{Appendix}

\subsection{Proof of Lemma 2.4}

Let $\left\{U_{\alpha}\right\}_{\alpha \in A}$ and $\left\{V_{\alpha}\right\}_{\alpha \in A}$ be finite coverings of $M$ with $\bar{U}_{\alpha} \subset V_{\alpha}$, such that there is an orthonormal basis $\left\{X_{i}^{\alpha}\right\}_{i=1, \ldots, n}$ of vector-fields over $V_{\alpha}$. Let $R$ be the constant from Lemma 2.2, then we define for every $\alpha$

$$
\begin{aligned}
& K_{\alpha}:=\left\{(x, y) \in M^{2}: x \in \bar{U}_{\alpha}, d_{g}(x, y) \leq R\right\} \\
& \tilde{K}_{\alpha}:=\left\{(x, y) \in M^{2}: x \in V_{\alpha}, d_{g}(x, y)<2 R\right\}
\end{aligned}
$$

and observe that each $K_{\alpha}$ is compact. Furthermore we define for $\alpha \in A, i=1, \ldots, n$

$$
h_{i}^{\alpha}: \tilde{K}_{\alpha} \longrightarrow T M \quad(x, y) \mapsto\left(y,\left(T \exp _{x}\right)_{\exp _{x}^{-1}(y)}\left(X_{i}^{\alpha}(x)\right)\right)
$$

which is smooth as a function of $(x, y)$. Hence its norm is bounded on $K_{\alpha}$, which proves (2.11) because there are only finitely many $\alpha \in A$. For fixed $x \in V_{\alpha}$ the map $h_{i}^{\alpha}(x, \cdot)$ is a vector-field on $B_{2 R}(x)$ and hence $\nabla_{y} h_{i}^{\alpha}(x, y)$, the covariant derivative with respect to $y$, is well-defined and smooth on $\tilde{K}_{\alpha}$. Furthermore

$$
\nabla_{y} h_{i}^{\alpha}(x, x)=0 \quad \forall x \in V_{\alpha}
$$

(cf. [13]), which implies

$$
\operatorname{div}_{g, y} h_{i}^{\alpha}(x, x)=0,
$$

because the divergence is the trace of the covariant derivative. For every $\alpha, i, j, k$

$$
(x, y) \mapsto\left\langle\nabla_{X_{j}(y)} h_{i}^{\alpha}(x, y), X_{k}(y)\right\rangle
$$

is smooth on $\tilde{K}_{\alpha}$ and vanishes for $x=y$ due to (6.3). Its gradient is bounded on $K_{\alpha}$ and we have

$$
\left\|\nabla_{y} h_{i}^{\alpha}(x, y)\right\|_{g} \leq d^{2} \sup _{j, k}\left|\left\langle\nabla_{X_{j}} h_{i}^{\alpha}, X_{k}\right\rangle\right| \leq d^{2} C_{i, \alpha} d_{g}(x, y)
$$

for $(x, y) \in K_{\alpha}$, where $\|\cdot\|_{g}$ denotes the operator norm with respect to $g$. This obviously implies

$$
\left|\operatorname{div}_{g, y} h_{i}^{\alpha}(x, y)\right| \leq d^{3} C_{i, \alpha} d_{g}(x, y) .
$$


Now let $x \in M, y \in B_{R}(x)$ and $v \in T_{x} M$ then there is some $\alpha \in A$ such that $x \in U_{\alpha}$ and we have

$$
v=\sum_{i}\left\langle X_{i}^{\alpha}(x), v\right\rangle X_{i}^{\alpha}(x)
$$

Hence

$$
\begin{aligned}
\left(T \exp _{x}\right)_{\exp _{x}^{-1}(y)}(v) & =\left(T \exp _{x}\right)_{\exp _{x}^{-1}(y)}\left(\sum_{i}\left\langle X_{i}^{\alpha}, v\right\rangle X_{i}^{\alpha}\right) \\
& =\sum_{i}\left\langle X_{i}^{\alpha}(x), v\right\rangle\left(T \exp _{x}\right)_{\exp _{x}^{-1}(y)}\left(X_{i}^{\alpha}(x)\right) \\
& =\sum_{i}\left\langle X_{i}^{\alpha}(x), v\right\rangle h_{i}^{\alpha}(x, y) \\
\Longrightarrow\left|\operatorname{div}_{g, y}\left(T \exp _{x}\right)_{\exp _{x}^{-1}(y)}(v)\right| & =\left|\sum_{i}\left\langle X_{i}^{\alpha}(x), v\right\rangle \operatorname{div}_{g, y} h_{i}^{\alpha}(x, y)\right| \\
& \leq \sum_{i}\left\langle X_{i}^{\alpha}(x), v\right\rangle d^{3} C_{i, \alpha} d_{g}(x, y) \\
& \leq\|v\|_{g} C d_{g}(x, y),
\end{aligned}
$$

where $C=d^{3} \sum_{i, \alpha} C_{i, \alpha}$. So we proved (2.10) and next we will show (2.12). Due to the continuity of $\chi^{\prime}$ we have

$$
\left|\chi^{\prime}\left(\frac{d_{g}(x, y)}{\varepsilon}\right)\right| \leq C 1_{\left\{d_{g}(x, y)<\varepsilon\right\}}
$$

and define

$$
\beta(t):=\left\langle\left(T \exp _{x}\right)_{\exp _{x}^{-1}\left(\gamma_{x y}(t)\right)}(v)-P_{x \gamma_{x y}(t)}(v), \gamma_{x y}^{\prime}(t)\right\rangle .
$$

We recall (2.9), hence to prove (2.12) we need an estimate for $\beta\left(d_{g}(x, y)\right)$. We have $\beta(0)=0$ and

$$
\begin{aligned}
\frac{\mathrm{d}}{\mathrm{d} t} \beta(t) & =\gamma_{x y}^{\prime}(t)\left\langle\left(T \exp _{x}\right)_{\exp _{x}^{-1}\left(\gamma_{x y}(t)\right)}(v)-P_{x \gamma_{x y}(t)}(v), \gamma_{x y}^{\prime}(t)\right\rangle \\
& =\left\langle\nabla_{\gamma_{x y}^{\prime}(t)}\left(T \exp _{x}\right)_{\exp _{x}^{-1}\left(\gamma_{x y}(t)\right)}(v), \gamma_{x y}^{\prime}(t)\right\rangle
\end{aligned}
$$

because $\nabla_{\gamma_{x y}^{\prime}} \gamma_{x y}^{\prime}=\nabla_{\gamma_{x y}^{\prime}} P_{x \gamma_{x y}}(v)=0$. Hence we have for some $\alpha \in A$

$$
\left|\frac{\mathrm{d}}{\mathrm{d} t} \beta(t)\right| \leq \sum_{i}\left\|\gamma_{x y}^{\prime}\right\|_{g}^{2}\left\|\nabla_{g, y} h_{i}^{\alpha}(x, y)\right\|_{g}\|v\|_{g} \stackrel{(6.4)}{\leq} C d_{g}(x, y)\|v\|_{g} .
$$

Hence we have $\left|\beta\left(d_{g}(x, y)\right)\right| \leq C\left(d_{g}(x, y)\right)^{2}\|v\|_{g}$ and using (2.9) and (6.5)

$$
\begin{aligned}
\left|\left\langle\left(T \exp _{x}\right)_{\exp _{x}^{-1}(y)}(v)-P_{x y}(v), \nabla_{g, y} \chi_{\varepsilon}(x, y)\right\rangle\right| & \leq \frac{1}{\varepsilon^{d+1}} C 1_{\left\{d_{g}(x, y)<\varepsilon\right\}}\left|\beta\left(d_{g}(x, y)\right)\right| \\
& \leq \frac{1}{\varepsilon^{d-1}} C 1_{\left\{d_{g}(x, y)<\varepsilon\right\}}\|v\|_{g} .
\end{aligned}
$$




\subsection{Proof of Lemma 3.2}

Proof. Miranda et al. showed in [16] that there exists a sequence $\left(f_{j}\right)_{j} \in C^{\infty}(M)$ such that

$$
\left\|f_{j}-u\right\|_{L^{1}(M)} \leq \frac{1}{j} \quad \text { and } \quad \lim _{j \rightarrow \infty} \int_{M}\left\|\nabla_{g} f_{j}\right\|_{g} \mathrm{dv}_{g}(x)=\operatorname{TV}_{M}(u)<\infty
$$

For every $j$ we have

$$
\begin{aligned}
\|u-\bar{u}\|_{L^{1}(M)} & \leq\left\|u-f_{j}\right\|_{L^{1}(M)}+\left\|f_{j}-\bar{f}_{j}\right\|_{L^{1}(M)}+\left\|\bar{f}_{j}-\bar{u}\right\|_{L^{1}(M)} \\
\left\|\overline{f_{j}}-\bar{u}\right\|_{L^{1}(M)} & =\sum_{K}\left\|\bar{f}_{j}-\bar{u}\right\|_{L^{1}(K)} \leq \sum_{K}\left\|f_{j}-u\right\|_{L^{1}(K)}=\left\|f_{j}-u\right\|_{L^{1}(M)} \\
\Rightarrow\|u-\bar{u}\|_{L^{1}(M)} & \leq 2\left\|u-f_{j}\right\|_{L^{1}(M)}+\left\|f_{j}-\bar{f}_{j}\right\|_{L^{1}(M)} .
\end{aligned}
$$

Furthermore we have for every $K \in \mathcal{T}$

$$
\begin{aligned}
\left\|f_{j}-\bar{f}_{j}\right\|_{L^{1}(K)} & \leq \int_{K}\left|f_{j}(x)-\frac{1}{|K|} \int_{K} f_{j}(y) \operatorname{dv}_{g}(y)\right| \operatorname{dv}_{g}(x) \\
& \leq \frac{1}{|K|} \int_{K^{2}}\left|f_{j}(x)-f_{j}(y)\right| \operatorname{dv}_{g}(y) \operatorname{dv}_{g}(x) .
\end{aligned}
$$

When the diameter of all elements is smaller than $R$, then for every pair of points $x, y \in K$ there is a unique minimizing geodesic from $x$ to $y$. It can be written as

$$
\gamma:[0,1] \longrightarrow M \quad \theta \mapsto \begin{cases}\exp _{y}\left((1-\theta) \exp _{y}^{-1}(x)\right) & \text { for } 0 \leq \theta \leq \frac{1}{2} \\ \exp _{x}\left(\theta \exp _{x}^{-1}(y)\right) & \text { for } \frac{1}{2} \leq \theta \leq 1\end{cases}
$$

This implies

$$
\begin{aligned}
\left\|f_{j}-\bar{f}_{j}\right\|_{L^{1}(K)} \leq & \frac{1}{|K|} \int_{K^{2}} \int_{0}^{\frac{1}{2}} \mid \nabla_{g} f_{j}\left(\exp _{y}\left((1-\theta) \exp _{y}^{-1}(x)\right)\right) \\
& \times\left(T \exp _{y}\right)_{(1-\theta) \exp _{y}^{-1}(x)}\left(\exp _{y}^{-1}(x)\right) \mid \mathrm{d} \theta \operatorname{dv}_{g}(x) \mathrm{dv}_{g}(y) \\
& +\frac{1}{|K|} \int_{K^{2}} \int_{\frac{1}{2}}^{1} \mid \nabla_{g} f_{j}\left(\exp _{x}\left(\theta \exp _{x}^{-1}(y)\right)\right) \\
& \times\left(T \exp _{x}\right)_{\theta \exp _{x}^{-1}(y)}\left(\exp _{x}^{-1}(y)\right) \mid \mathrm{d} \theta \operatorname{dv}_{g}(x) \operatorname{dv}_{g}(y) .
\end{aligned}
$$

Due to (2.11) we have

$$
\left\|\left(T \exp _{y}\right)_{(1-\theta) \exp _{y}^{-1}(x)}\left(\exp _{y}^{-1}(x)\right)\right\|_{g} \leq C\left\|\exp _{y}^{-1}(x)\right\|_{g} \leq C \delta(K) .
$$


Inserting (6.8) in (6.7) implies

$$
\begin{aligned}
\left\|f_{j}-\bar{f}_{j}\right\|_{L^{1}(K)} \leq & \frac{C \delta(K)}{|K|} \int_{K^{2}} \int_{0}^{\frac{1}{2}}\left\|\nabla_{g} f_{j}\left(\exp _{y}\left((1-\theta) \exp _{y}^{-1}(x)\right)\right)\right\|_{g} \mathrm{~d} \theta \operatorname{dv}_{g}(x) \operatorname{dv}_{g}(y) \\
& +\frac{C \delta(K)}{|K|} \int_{K^{2}} \int_{\frac{1}{2}}^{1}\left\|\nabla_{g} f_{j}\left(\exp _{x}\left(\theta \exp _{x}^{-1}(y)\right)\right)\right\|_{g} \mathrm{~d} \theta \operatorname{dv}_{g}(x) \operatorname{dv}_{g}(y) \\
= & \frac{C \delta(K)}{|K|} \int_{K} \int_{0}^{\frac{1}{2}} \int_{(1-\theta) \exp _{y}^{-1}(K)}\left\|\nabla_{g} f_{j}\left(\exp _{y}(w)\right)\right\|_{g} \frac{1}{(1-\theta)^{d}}\left|\operatorname{det}\left(T \exp _{y}\right)_{w}\right| \mathrm{d} \theta \mathrm{d} w \operatorname{dv} v_{g}(y) \\
& +\frac{C \delta(K)}{|K|} \int_{K} \int_{\frac{1}{2}}^{1} \int_{\theta \exp _{x}^{-1}(K)}\left\|\nabla_{g} f_{j}\left(\exp _{x}(v)\right)\right\|_{g} \frac{1}{\theta^{d}}\left|\operatorname{det}\left(T \exp _{x}\right)_{v}\right| \mathrm{d} \theta \mathrm{d} v \operatorname{dv}_{g}(x)
\end{aligned}
$$

where the determinants are computed with respect to orthonormal bases of the respective tangent spaces. The determinant of $\left(T \exp _{x}\right)_{v}$ is continuous and positive on the compact set $\mathcal{K}:=\left\{(x, v) \in T M:\|v\|_{g} \leq R\right\}$ so there exists $C>0$ such that

$$
\frac{1}{C}<\left|\operatorname{det}\left(T \exp _{x}\right)_{v}\right|<C \quad \forall(x, v) \in \mathcal{K} .
$$

We have

$$
\begin{aligned}
\left\|f_{j}-\bar{f}_{j}\right\|_{L^{1}(K)} \leq & \frac{C \delta(K)}{|K|} \int_{K} \int_{0}^{\frac{1}{2}} \int_{\exp _{y}\left((1-\theta) \exp _{y}^{-1}(K)\right)}\left\|\nabla_{g} f_{j}(z)\right\|_{g}\left|\operatorname{det}\left(\left(T \exp _{y}^{-1}\right)_{z}\right)\right| \operatorname{dv}_{g}(z) \mathrm{d} \theta \operatorname{dv}_{g}(y) \\
& +\frac{C \delta(K)}{|K|} \int_{K} \int_{\frac{1}{2}}^{1} \int_{\exp _{x}\left(\theta \exp _{x}^{-1}(K)\right)}\left\|\nabla_{g} f_{j}(z)\right\|_{g}\left|\operatorname{det}\left(\left(T \exp _{x}^{-1}\right)_{z}\right)\right| \operatorname{dv}_{g}(z) \mathrm{d} \theta \operatorname{dv}_{g}(x) .
\end{aligned}
$$

By definition of convex hull (Def. 2.3) we get

$$
\begin{array}{r}
\exp _{y}\left((1-\theta) \exp _{y}^{-1}(K)\right) \subset \operatorname{conv} K \text { for } 0 \leq \theta \leq 1 \text { and } x, y \in K \\
\exp _{x}\left(\theta \exp _{x}^{-1}(K)\right) \subset \operatorname{conv} K \text { for } 0 \leq \theta \leq 1 \text { and } x, y \in K
\end{array}
$$

This implies by (6.9) due to

$$
\begin{aligned}
\left\|f_{j}-\bar{f}_{j}\right\|_{L^{1}(K)} & \leq \frac{C \delta(K)}{|K|} \int_{K} \int_{\operatorname{conv} K}\left\|\nabla_{g} f_{j}(z)\right\|_{g} \operatorname{dv}_{g}(z) \operatorname{dv}_{g}(y)+\frac{C \delta(K)}{|K|} \int_{K} \int_{\operatorname{conv} K}\left\|\nabla_{g} f_{j}(z)\right\|_{g} \operatorname{dv}_{g}(z) \operatorname{dv}_{g}(x) \\
& \leq C \delta(K)\left\|\nabla_{g} f_{j}\right\|_{L^{1}(\operatorname{conv} K)} .
\end{aligned}
$$

Finally we have due to (6.6) and our assumption on the triangulation

$$
\begin{aligned}
\|u-\bar{u}\|_{L^{1}(M)} & \leq \lim _{j \rightarrow \infty} 2\left\|u-f_{j}\right\|_{L^{1}(M)}+\lim _{j \rightarrow \infty}\left\|f_{j}-\bar{f}_{j}\right\|_{L^{1}(M)} \\
& =\lim _{j \rightarrow \infty} \sum_{K}\left\|f_{j}-\bar{f}_{j}\right\|_{L^{1}(K)} \\
& \leq C h \lim _{j \rightarrow \infty} \sum_{K}\left\|\nabla_{g} f_{j}\right\|_{L^{1}(\operatorname{conv} K)} \\
& \leq C h N_{c} T V_{M}(u) .
\end{aligned}
$$




\section{REFERENCES}

[1] P. Amorim, M. Ben-Artzi and P.G. LeFloch, Hyperbolic conservation laws on manifolds: total variation estimates and the finite volume method. Methods Appl. Anal. 12 (2005) 291-323.

[2] M. Ben-Artzi and P.G. LeFloch, Well-posedness theory for geometry compatible hyperbolic conservation laws on manifolds. Ann. H. Poincaré Anal. Non Linéaire 24 (2007) 989-1008.

[3] D.A. Calhoun, C. Helzel and R.J. LeVeque, Logically rectangular grids and finite volume methods for PDEs in circular and spherical domains. SIAM Rev. 50 (2008) 723-752. Available at http://www.amath.washington.edu/ rjl/pubs/circles.

[4] J.Y.-K. Cho and L.M. Polvani, The emergence of jets and vortices in freely evolving, shallow-water turbulence on a sphere. Phys. Fluids 8 (1996) 1531-1552.

[5] M. Dikpati and P.A. Gilman, A "shallow-water" theory for the sun's active longitudes. Astrophys. J. Lett. 635 (2005) L193L196.

[6] M.P. do Carmo, Riemannian geometry, Mathematics: Theory \& Applications. Birkhäuser Boston Inc., Boston, USA (1992).

[7] R. Eymard, T. Gallouët, M. Ghilani and R. Herbin, Error estimates for the approximate solutions of a nonlinear hyperbolic equation given by finite volume schemes. IMA J. Numer. Anal. 18 (1998) 563-594.

[8] J.A. Font, Numerical hydrodynamics and magnetohydrodynamics in general relativity. Living Rev. Relativ. 11 (2008) 7. URL (cited on June 8, 2009): http://www.livingreviews.org/lrr-2008-7.

[9] P.A. Gilman, Magnetohydrodynamic "shallow-water" equations for the solar tachocline. Astrophys. J. Lett. 544 (2000) L79L82.

[10] F.X. Giraldo, Lagrange-Galerkin methods on spherical geodesic grids. J. Comput. Phys. 136 (1997) 197-213.

[11] F.X. Giraldo, High-order triangle-based discontinuous Galerkin methods for hyperbolic equations on a rotating sphere. J. Comput. Phys. 214 (2006) 447-465.

[12] R. Iacono, M.V. Struglia and C. Ronchi, Spontaneous formation of equatorial jets in freely decaying shallow water turbulence. Phys. Fluids 11 (1999) 1272-1274.

[13] J. Jost, Riemannian Geometry and Geometric Analysis. Springer Universitext, Springer (2002).

[14] D. Lanser, J.G. Blom and J.G. Verwer, Spatial discretization of the shallow water equations in spherical geometry using osher's scheme. J. Comput. Phys. 165 (2000) 542-565.

[15] J.M. Martí and E. Müller, Numerical hydrodynamics in special relativity. Living Rev. Relativ. 6 (2003) 7. URL (cited on June 8, 2009): http://www.livingreviews.org/lrr-2003-7.

[16] M.J. Miranda, D. Pallara, F. Paronetto and M. Preunkert, Heat semigroup and functions of bounded variation on Riemannian manifolds. J. reine angew. Math. 613 (2007) 99-119.

[17] M. Rancic, R.J. Purser and F. Mesinger, A global shallow-water model using an expanded spherical cube: Gnomonic versus conformal coordinates. Q. J. R. Meteorolog. Soc. 122 (1996) 959-982.

[18] C. Ronchi, R. Iacono and P.S. Paolucci, The cubed sphere: A new method for the solution of partial differential equations in spherical geometry. J. Comput. Phys. 124 (1996) 93-114.

[19] J.A. Rossmanith, A wave propagation algorithm for hyperbolic systems on the sphere. J. Comput. Phys. 213 (2006) 629-658.

[20] J.A. Rossmanith, D.S. Bale and R.J. LeVeque, A wave propagation algorithm for hyperbolic systems on curved manifolds. J. Comput. Phys. 199 (2004) 631-662.

[21] D.A. Schecter, J.F. Boyd and P.A. Gilman, "Shallow-water" magnetohydrodynamic waves in the solar tachocline. Astrophys. J. Lett. 551 (2001) L185-L188.

[22] Y. Tsukahara, N. Nakaso, H. Cho and K. Yamanaka, Observation of diffraction-free propagation of surface acoustic waves around a homogeneous isotropic solid sphere. Appl. Phys. Lett. 77 (2000) 2926-2928. 